JOURNAL OF THE AMERICAN MATHEMATICAL SOCIETY

Volume 10, Number 1, January 1997, Pages 215-242

S 0894-0347(97)00221-X

\title{
THE CLASSIFICATION OF HYPERSMOOTH BOREL EQUIVALENCE RELATIONS
}

\author{
ALEXANDER S. KECHRIS AND ALAIN LOUVEAU
}

This paper is a contribution to the study of Borel equivalence relations in standard Borel spaces, i.e., Polish spaces equipped with their Borel structure. A class of such equivalence relations which has received particular attention is the class of hyperfinite Borel equivalence relations. These can be defined as the increasing unions of sequences of Borel equivalence relations all of whose equivalence classes are finite or, as it turns out, equivalently those induced by the orbits of a single Borel automorphism. Hyperfinite equivalence relations have been classified in [DJK], under two notions of equivalence, Borel bi-reducibility, and Borel isomorphism.

An equivalence relation $E$ on $X$ is Borel reducible to an equivalence relation $F$ on $Y$ if there is a Borel map $f: X \rightarrow Y$ with $x E y \Leftrightarrow f(x) F f(y)$. We write then $E \leq F$. If $E \leq F$ and $F \leq E$ we say that $E, F$ are Borel bi-reducible, in symbols $E \approx{ }^{*} F$. When $E \approx^{*} F$ the quotient spaces $X / E, Y / F$ have the same "effective" or "definable" cardinality. We say that $E, F$ are Borel isomorphic if there exists a Borel bijection $f: X \rightarrow Y$ with $x E y \Leftrightarrow f(x) F f(y)$. Below we denote by $E_{0}, E_{t}$ the equivalence relations on the Cantor space $2^{\mathbb{N}}$ given by: $x E_{0} y \Leftrightarrow$ $\exists n \forall m \geq n\left(x_{m}=y_{m}\right), x E_{t} y \Leftrightarrow \exists n \exists k \forall m\left(x_{n+m}=y_{k+m}\right)$. We denote by $\Delta_{X}$ the equality relation on $X$, and finally we call $E$ smooth if $E \leq \Delta_{2^{\mathbb{N}}}$. This just means that elements of $X$ can be classified up to $E$-equivalence by concrete invariants which are members of some Polish space.

It is shown now in [DJK] that up to Borel bi-reducibility there is exactly one non-smooth hyperfinite Borel $E$, namely $E_{0}$, and up to Borel isomorphism there are exactly countably many non-smooth hyperfinite aperiodic (i.e., having no finite equivalence classes) Borel $E$, namely $E_{t}, E_{0} \times \Delta_{n}\left(1 \leq n \leq \aleph_{0}\right), E_{0} \times \Delta_{2^{\mathbb{N}}}$ (where $\Delta_{n}=\Delta_{X}$, with $\operatorname{card}(X)=n$, if $\left.1 \leq n \leq \aleph_{0}\right)$.

In this paper we investigate and classify the class of Borel equivalence relations which are the "continuous" analogs of the hyperfinite ones. We call a Borel equivalence relation $E$ hypersmooth if it can be written as $E=\bigcup_{n} E_{n}$, where $E_{0} \subseteq E_{1} \subseteq \cdots$ is an increasing sequence of smooth Borel equivalence relations. These have been also studied (in a measure theoretic context) in the Russian literature under the name tame equivalence relations. They include many interesting examples such as: The increasing union of a sequence of closed or even $G_{\delta}$ equivalence relations (like for example the coset equivalence relation of a Polish group modulo a subgroup, which is the increasing union of a sequence of closed subgroups), the hyperfinite equivalence relations, the "tail" equivalence relations

Received by the editors September 1, 1994 and, in revised form, June 11, 1996.

1991 Mathematics Subject Classification. Primary 04A15, 03E15.

Key words and phrases. Borel equivalence relations, hypersmooth, dichotomy theorems.

The first author's research was partially supported by NSF Grant DMS-9317509. 
$E_{0}(U), E_{t}(U)$ of a Borel map $U: X \rightarrow X$ given by $x E_{0}(U) y \Leftrightarrow \exists n\left(U^{n}(x)=U^{n}(y)\right)$ and $x E_{t}(U) y \Leftrightarrow \exists n \exists m\left(U^{n}(x)=U^{m}(y)\right)$, the equivalence relations induced by the orbits of a Borel action of a Polish locally compact group which is compactly generated of polynomial growth (e.g., $\mathbb{R}^{n}$ ), the equivalence relation induced by the composants of an indecomposable continuum, etc.

Denote by $E_{1}$ the equivalence relation on $\left(2^{\mathbb{N}}\right)^{\mathbb{N}}$ given by $x E_{1} y \Leftrightarrow \exists n \forall m \geq n$ $\left(x_{m}=y_{m}\right)$. This is the "continuous" analog of $E_{0}$ and is clearly hypersmooth. It is well-known that $E_{0}<E_{1}$ (i.e., $E_{0} \leq E_{1}$, but $\left.E_{1} \not \leq E_{0}\right)$ and it is easy to see that $E \leq E_{1}$ for any Borel hypersmooth $E$. The main result in this paper is now the following dichotomy, which was motivated by results in the measure theoretic context, see [V], [VF], [VG].

Theorem 1. If $E$ is a hypersmooth Borel equivalence relation, then exactly one of the following holds:

(I) $E \leq E_{0}$;

(II) $E_{1} \leq E$.

(Actually in (II) the reducing function can be taken to be injective, i.e., an embedding.)

From this it follows that up to Borel bi-reducibility there are exactly two nonsmooth hypersmooth Borel equivalence relations, namely $E_{0}$ and $E_{1}$. With some further work one can obtain also results on classification up to Borel isomorphism. For example, up to Borel isomorphism there are only two non-smooth hypersmooth Borel $E$, satisfying some mild natural conditions, that have equivalence classes of size $2^{\aleph_{0}}$, namely $E_{0} \times I_{2^{\mathbb{N}}}$ and $E_{1}\left(\right.$ where $I_{2^{\mathbb{N}}}=2^{\mathbb{N}} \times 2^{\mathbb{N}}$ ).

Despite the fact that our main result involves only notions of classical descriptive set theory, the proof makes heavy use of effective descriptive set theory, as was the case with the proof of the Glimm-Effros type dichotomy for Borel equivalence relations proved in [HKL].

Although the dichotomy expressed in Theorem 1 is of a "local" nature, as it refers only to hypersmooth Borel equivalence relations, it turns out surprisingly to have also global consequences concerning the structure of arbitrary Borel equivalence relations. Consider the partial (pre-)order $\leq$ on Borel equivalence relations. A node is a Borel equivalence relation $E$ such that for any Borel $F, E \leq F$ or $F \leq E$, i.e., $E$ is comparable to any Borel equivalence relation. It is trivial that each $\Delta_{n}$ $(n=1,2, \ldots)$ is a node and by Silver's Theorem in $[\mathrm{S}]$, which implies that for any Borel $E$ either $E \leq \Delta_{\aleph_{0}}$ or $\Delta_{2^{\mathbb{N}}} \leq E$, we have that $\Delta_{\aleph_{0}}, \Delta_{2^{\mathbb{N}}}$ are also nodes. We now have:

Theorem 2. The only nodes in the partial order $\leq$ on Borel equivalence relations are $\Delta_{n}\left(1 \leq n \leq \aleph_{0}\right), \Delta_{2^{\mathbb{N}}}$, and $E_{0}$.

This has the following immediate implication. Say that a pair of Borel equivalence relations $\left(E, E^{*}\right)$ with $E<E^{*}$ has the dichotomy property if for any Borel equivalence relation $F$ we have $F \leq E$ or $E^{*} \leq F$. Clearly $\left(\Delta_{n}, \Delta_{n+1}\right)$, $n=1,2, \ldots$, have this property. By Silver's Theorem so does $\left(\Delta_{\aleph_{0}}, \Delta_{2^{\mathbb{N}}}\right)$, and by the result in [HKL] the same holds for $\left(\Delta_{2^{\mathbb{N}}}, E_{0}\right)$. It follows from Theorem 2 that these are the only such pairs, i.e., except for the trivial case of $\left(\Delta_{n}, \Delta_{n+1}\right)$, the only global dichotomy theorems for Borel equivalence relations are Silver's Theorem and the general Glimm-Effros Dichotomy established in [HKL]. 
The paper is organized as follows: Section 0 contains preliminaries on descriptive set theory and equivalence relations. Section 1 discusses the basic properties of hypersmooth relations and several examples. In Section 2 we prove the main theorem. Section 3 contains consequences concerning isomorphism classifications. In Section 4 we discuss results and examples relating to the possibility of reducing $E_{1}$ to other Borel equivalence relations. Finally, Section 5 contains the "global" consequences of our main results mentioned above.

\section{Preliminaries}

A) A standard Borel space is a set $X$ equipped with a $\sigma$-algebra $\mathcal{S}$ such that for some Polish (i.e., separable completely metrizable) topology $\tau$ on $X, \mathcal{S}$ in the class of Borel sets of $\tau$. We call the members of $\mathcal{S}$ the Borel sets in $X$. Every uncountable standard Borel space is Borel isomorphic to the Baire space $\mathcal{N}=\mathbb{N}^{\mathbb{N}}$ and to the Cantor space $\mathcal{C}=2^{\mathbb{N}}$.

We use the customary notation and terminology concerning descriptive set theory, see, e.g., [Mo]. In particular $\boldsymbol{\Sigma}_{1}^{1}$ denotes the class of analytic sets, $\boldsymbol{\Pi}_{1}^{1}$ the class of co-analytic sets and $\boldsymbol{\Delta}_{1}^{1}$ the class of bi-analytic sets, i.e., these which are both analytic and co-analytic. By Souslin's Theorem the bi-analytic sets are exactly the Borel sets.

The use of effective descriptive set theory is crucial for the proof of our main result. Again we use standard terminology and notation as in [Mo]. Thus $\Sigma_{1}^{1}, \Pi_{1}^{1}, \Delta_{1}^{1}$ denote resp. the classes of effectively analytic, co-analytic and bi-analytic sets. We denote by $\omega_{1}^{x}$ the first ordinal not recursive in $x$ and by $\omega_{1}^{\mathrm{CK}}$ the first non-recursive ordinal.

The results from (both classical and effective) descriptive set theory that we will use can be found in $[\mathrm{Mo}]$, and in [HKL] in regards to the Gandy-Harrington topology, with the exception of two reflection theorems that we will now state. Their proofs can be found in [HMS], [K3].

0.1. First Reflection Theorem. Let $\Phi \subseteq \mathcal{P}(\mathcal{N})(=$ the power set of $\mathcal{N})$ be $\Pi_{1}^{1}$ on $\Sigma_{1}^{1}$, i.e., for $B \subseteq \mathcal{N} \times \mathcal{N}$ in $\Sigma_{1}^{1},\left\{y: \Phi\left(B_{y}\right)\right\}$ is in $\Pi_{1}^{1}$. Then if $\Phi(A)$ holds for $A \in \Sigma_{1}^{1}$, there is $A^{\prime} \supseteq A, A^{\prime} \in \Delta_{1}^{1}$ such that $\Phi\left(A^{\prime}\right)$ holds.

0.2. Burgess Reflection Theorem. Let $R \subseteq \mathcal{N}^{\mathbb{N}} \times \mathcal{N}^{n}(n \in \mathbb{N})$ be $\Pi_{1}^{1}$ and let $\Phi \subseteq \mathcal{P}(\mathcal{N})$ be given by

$$
\begin{aligned}
\Phi(A) \Leftrightarrow \forall x \in \mathcal{N}^{\mathbb{N}} \forall y \in \mathcal{N}^{n} & \left\{n\left(x_{n}\right) \in A\right) \& \\
& \left.\forall i<n\left(y_{i} \notin A\right) \Rightarrow R(x, y)\right\} .
\end{aligned}
$$

If $A \subseteq \mathcal{N}$ is $\Sigma_{1}^{1}$ and $\Phi(A)$ holds, then there is $A^{\prime} \supseteq A, A^{\prime} \in \Delta_{1}^{1}$ such that $\Phi\left(A^{\prime}\right)$ holds.

B) By a Polish group we mean a topological group whose topology is Polish. If $X$ is a standard Borel space, a Borel action of $G$ on $X$ is an action $(g, x) \mapsto g \cdot x$ of $G$ on $X$ which is Borel as a function from $G \times X$ into $X$.

C) If $X$ is a set and $E$ an equivalence relation on $X$, we denote by $[x]_{E}$ the equivalence class of $x$, by $X / E=\left\{[x]_{E}: x \in X\right\}$ the quotient space of $X$ by $E$, and by $[A]_{E}=\{x: \exists y \in A(x E y)\}$ the $E$-saturation of $A \subseteq X$. If $[A]_{E}=A$ we say that $A$ is $E$-invariant. 
A transversal for $E$ is a subset $T \subseteq X$ which meets every equivalence class in exactly one point. A selector for $E$ is a map $s: X \rightarrow X$ with $x E y \Rightarrow s(x)=$ $s(y) E y$.

We denote by $\Delta_{X}, I_{X}$ respectively the smallest and largest equivalence relations on $X$, i.e., $\Delta_{X}$ is equality on $X$ and $I_{X}=X^{2}$.

If $A \subseteq X$, we denote by $E \mid A$ the restriction of $E$ to $A$, i.e., $E \mid A=E \cap A^{2}$. If $F$ is also an equivalence relation on $X, E \subseteq F$ means that $E$ is a subequivalence relation of $F$, i.e., $x E y \Rightarrow x F y$.

Suppose now $E, F$ are equivalence relations on $X, Y$ resp. A reduction of $E$ into $F$ is a map $f: X \rightarrow Y$ with $x E y \Leftrightarrow f(x) F f(y)$. Note that this induces an injection $f^{*}: X / E \rightarrow Y / F$ given by $f^{*}\left([x]_{E}\right)=[f(x)]_{F}$. If $f$ is $1-1$ we call this an embedding. If $f$ is $1-1$ and onto it is called an isomorphism of $E, F$. If $f$ is an embedding and $f[X]=B$ is $F$-invariant, then we say that it is an invariant embedding. It is clearly an isomorphism of $E$ with $F \mid B$. Invariant embeddings of $E$ into $F$ and $F$ into $E$ give rise, via the standard Schroeder-Bernstein argument, to an isomorphism of $E$ and $F$.

The product of $E, F$ is the equivalence relation $E \times F$ on $X \times Y$ defined by

$$
(x, y) E \times F\left(x^{\prime}, y^{\prime}\right) \Leftrightarrow x E x^{\prime} \& y F y^{\prime} .
$$

D) Assume now $E, F$ are equivalence relations on standard Borel spaces $X, Y$. We write

$$
\begin{aligned}
& E \leq F \quad \Leftrightarrow \exists \text { a Borel reduction of } E \text { into } F, \\
& E<F \quad \Leftrightarrow E \leq F \& F \not \leq E ; \\
& E \approx^{*} F \Leftrightarrow E \leq F \& F \leq E ; \\
& E \sqsubseteq F \quad \Leftrightarrow \exists \text { a Borel embedding of } E \text { into } F ; \\
& E \approx F \Leftrightarrow E \sqsubseteq F \& F \sqsubseteq E ; \\
& E \sqsubseteq^{i} F \Leftrightarrow \exists \text { a Borel invariant embedding of } E \text { into } F ; \\
& E \cong F \quad \Leftrightarrow \exists \text { a Borel isomorphism of } E, F .
\end{aligned}
$$

Note that

$$
E \cong F \Leftrightarrow E \sqsubseteq^{i} F \& F \sqsubseteq^{i} E .
$$

Now let $E$ be a Borel equivalence relation on a standard Borel space $X$. We call $E$ smooth if $E$ has a countable Borel separating family, i.e., a sequence $\left(A_{n}\right)$ of Borel sets in $X$ with

$$
x E y \Leftrightarrow \forall n\left(x \in A_{n} \Leftrightarrow y \in A_{n}\right) .
$$

This is easily equivalent to saying that $E \leq \Delta_{X}$, for some standard Borel space $X$. If $E$ admits a Borel transversal (equivalently a Borel selector), then $E$ is smooth. The converse is in general false (see, e.g., [K3, 18.D]), but holds for most natural examples.

The following dichotomy result was proved in [HKL]. Let $E_{0}$ be the equivalence relation on $2^{\mathbb{N}}$ given by

$$
x E_{0} y \Leftrightarrow \exists n \forall m \geq n\left(x_{m}=y_{m}\right) .
$$

Then for any Borel $E$, exactly one of the following holds: $E$ is smooth or $E_{0} \sqsubseteq E$. In fact the following effective version is proved in [HKL]: If $E$ is a $\Delta_{1}^{1}$ equivalence relation on $\mathcal{N}$, then exactly one of the following holds: $E \leq \Delta_{2^{\mathbb{N}}}$ via a $\Delta_{1}^{1}$ reduction or $E_{0} \sqsubseteq E$. 
E) A Borel equivalence relation $E$ on $X$ is called finite, resp. countable, if every equivalence class $[x]_{E}$ is finite, resp. countable. It is called hyperfinite if $E=\bigcup_{n} E_{n}$, with $E_{0} \subseteq E_{1} \subseteq \cdots$ an increasing sequence of finite Borel equivalence relations. Clearly hyperfinite equivalence relations are countable. For more about their structure, see [DJK]. For example, they can be characterized as those that are induced by the orbits of a Borel action of $\mathbb{Z}$ on $X$, i.e., which are of the form $E=\left\{\left(x, T^{n}(x)\right): n \in \mathbb{Z}\right\}$ with $T$ a Borel automorphism of $X$. Also they turn out to be exactly those that can be written as $E=\bigcup_{n} E_{n}$, with $E_{0} \subseteq E_{1} \subseteq \cdots$ an increasing sequence of smooth countable Borel equivalence relations.

\section{BASIC FACTS AND EXAMPLES}

Let $X$ be a standard Borel space and $E$ a Borel equivalence relation on $X$. We call $E$ hypersmooth if $E=\bigcup_{n} F_{n}$, where $F_{0} \subseteq F_{1} \subseteq F_{2} \subseteq \cdots$ is an increasing sequence of smooth Borel equivalence relations. Such equivalence relations are called tame in the Russian literature; see $[\mathrm{V}],[\mathrm{VF}],[\mathrm{VG}]$.

Let us note some simple closure properties of hypersmooth relations.

Proposition 1.1. (i) If $F$ is hypersmooth and $E \leq F$, then $E$ is hypersmooth;

(ii) If $E$ is hypersmooth and $A$ is Borel, $E \mid A$ is hypersmooth;

(iii) If $E, F$ are hypersmooth, so is $E \times F$.

The proofs are straightforward.

The following is a basic open problem.

Problem 1.2. If $E=\bigcup_{n} F_{n}$, where $F_{0} \subseteq F_{1} \subseteq \cdots$ is an increasing sequence of Borel hypersmooth equivalence relations, is $E$ hypersmooth?

We next discuss examples:

$0)$ It is well-known (see, e.g., [K1, 2.2]) that ever closed equivalence relation is smooth, and in [HKL] this is extended to $G_{\delta}$ equivalence relations. So if $E=\bigcup_{n} E_{n}$, $E_{0} \subseteq E_{1} \subseteq \cdots$ an increasing sequence of closed or even $G_{\delta}$ equivalence relations, then $E$ is hypersmooth. Conversely, it follows from [K3, 13.11] that if $E$ is Borel hypersmooth on the standard Borel space $X$, there is a Polish topology $\tau$ giving the Borel structure of $X$, such that $E=\bigcup_{n} E_{n}$, with $E_{0} \subseteq E_{1} \subseteq \cdots$ closed in $\left(X^{2}, \tau^{2}\right)$ equivalence relations.

1) Every Borel hyperfinite equivalence relation (see [DJK]) is hypersmooth. In fact, we view hypersmooth relations as "continuous" analogs of the hyperfinite ones.

2 ) For any standard Borel space $\Omega$, let $E_{0}(\Omega), E_{t}(\Omega)$ be the following equivalence relations on $X=\Omega^{\mathbb{N}}$ :

$$
\begin{aligned}
& x E_{0}(\Omega) y \Leftrightarrow \exists n \forall m \geq n\left(x_{m}=y_{m}\right), \\
& x E_{t}(\Omega) y \Leftrightarrow \exists n \exists m \forall k\left(x_{n+k}=y_{m+k}\right) .
\end{aligned}
$$

It is clear that $E_{0}(\Omega)$ is hypersmooth, and it is shown in [DJK] that so is $E_{t}(\Omega)$.

Put

$$
\begin{aligned}
& E_{0}=E_{0}(2), \\
& E_{1}=E_{0}\left(2^{\mathbb{N}}\right) .
\end{aligned}
$$

3) We can generalize the examples in 2) as follows:

Let $X$ be a standard Borel space and $U: X \rightarrow X$ a Borel map. Put

$$
\begin{aligned}
& x E_{0}(U) y \Leftrightarrow \exists n\left(U^{n}(x)=U^{n}(y)\right), \\
& x E_{t}(U) y \Leftrightarrow \exists n \exists m\left(U^{n}(x)=U^{m}(y)\right) .
\end{aligned}
$$


Then $E_{0}(U), E_{t}(U)$ are hypersmooth (see [DJK]). If we take $X=\Omega^{\mathbb{N}}$ and $U\left(\left(x_{n}\right)\right)=$ $\left(x_{n+1}\right)$, the shift on $\Omega^{\mathbb{N}}$, we obtain the examples in 2$)$.

4) Let $G$ be a Polish group and $H \subseteq G$ a subgroup. Let $G / H=\{x H: x \in G\}$ be the (left) coset space of $H$ in $G$ and put

$$
x E_{H} y \Leftrightarrow x H=y H
$$

for the associated equivalence relation. If $H$ is closed, then it is well-known that $E_{H}$ is smooth, in fact has a Borel transversal. Conversely (see [Mi]), if $H$ is Borel and $E_{H}$ is smooth, then $H$ is closed.

If now $H=\bigcup_{n} H_{n}$, with $H_{0} \subseteq H_{1} \subseteq \cdots$ an increasing sequence of closed subgroups of $G$, then $E_{H}$ is clearly hypersmooth. Both $E_{0}, E_{1}$ are of this form. For $E_{0}$, we take $G=\mathbb{Z}_{2}^{\mathbb{N}}, H_{n}=\mathbb{Z}_{2}^{n}$ (viewed as a subgroup of $\mathbb{Z}_{2}^{\mathbb{N}}$ by identifying $\left(x_{1}, \ldots, x_{n}\right) \in \mathbb{Z}_{2}^{\mathbb{N}}$ with $\left.\left(x_{1}, x_{2}, \ldots, x_{n}, 0,0, \ldots\right)\right)$. For $E_{1}$ let $G=\mathbb{T}^{\mathbb{N}}$ (T) the unit circle), $H_{n}=\mathbb{T}^{n}$. (This does not give literally $E_{1}$, which lives on $2^{\mathbb{N}}$, but a Borel isomorphic copy of it.)

5) If $G$ is a Polish locally compact group and $(g, x) \mapsto g \cdot x$ a Borel action of $G$ on $X$, we denote by $E_{G}$ the (Borel) equivalence relation induced by the orbits of this action, i.e.,

$$
x E_{G} y \Leftrightarrow \exists g \in G(g \cdot x=y) .
$$

It is shown in $[\mathrm{W}]$ and $[\mathrm{K} 1]$ that $E_{\mathbb{R}} \leq E_{0}$, so $E_{\mathbb{R}}$ is hypersmooth. Thus the orbit equivalence relation of a flow (i.e., an $\mathbb{R}$-action) is hypersmooth. This was extended in [JKL] to show that $E_{G} \leq E_{0}$ for any $G$ which is compactly generated of polynomial growth (e.g., $\mathbb{R}^{n}$ ); thus all such $E_{G}$ are hypersmooth.

6 ) The following interesting example was discovered recently by Solecki: Let $X$ be a continuum (i.e., a compact connected metric space). It is called indecomposable if it is not the union of two proper subcontinua. For any indecomposable continuum $X$ and $x \in X$, the composant of $x$ is the union of all proper subcontinua containing $x$. The composants form a partition of $X$ (into $2^{\aleph_{0}}$ pieces), and let us denote by $E_{X}$ the corresponding equivalence relation. By a result of Rogers [R], $E_{X}$ is $F_{\sigma}$. Solecki has in fact shown that $E=\bigcup_{n} E_{n}, E_{0} \subseteq E_{1} \subseteq \cdots$ an increasing sequence of closed equivalence relations, so $E$ is hypersmooth.

The equivalence relation $E_{1}$ is universal among hypersmooth Borel equivalence relations.

Proposition 1.3. Let $E$ be a hypersmooth Borel equivalence relation. Then $E \sqsubseteq$ $E_{1}$.

Proof. Let $E=\bigcup_{n} F_{n}$, with $F_{n}$ an increasing sequence of smooth Borel equivalence relations on $X$. Let $f_{n}: X \rightarrow 2^{\mathbb{N}}$ be Borel with $x F_{n} y \Leftrightarrow f_{n}(x)=f_{n}(y)$ and assume that $F_{0}=\Delta_{X}$, so that $f_{0}$ is injective. Define $f: X \rightarrow\left(2^{\mathbb{N}}\right)^{\mathbb{N}}$ by

$$
f(x)=\left(f_{0}(x), f_{1}(x), \cdots\right) .
$$

Then $f$ is Borel injective and $x E y \Leftrightarrow f(x) E_{1} f(y)$, so $E \sqsubseteq E_{1}$.

The universal relation $E_{1}$ also has the following important property which has been known for some time (see, e.g., $[\mathrm{FR}],[\mathrm{K} 1, \S 5]$ ).

Proposition 1.4. If $F$ is a countable Borel equivalence relation, then $E_{1} \not \leq F$. In particular, $E_{0}<E_{1}$. 
In fact 1.4 is also a consequence of the following stronger result, which also has other applications.

Theorem 1.5. Let $X$ be a standard Borel space, $F$ a countable Borel equivalence relation on $X$ and $f:\left(2^{\mathbb{N}}\right)^{\mathbb{N}} \rightarrow X$ a Borel map such that $x E_{1} y \Rightarrow f(x) F f(y)$. Then there are $\left(x_{n}\right),\left(y_{n}\right) \in\left(2^{\mathbb{N}}\right)^{\mathbb{N}}$ with $n \mapsto x_{n}, n \mapsto y_{n}$ injective, $x_{n} \neq y_{m}$ for all $n, m$ and $f\left(\left(x_{n}\right)\right)=f\left(\left(y_{n}\right)\right)$.

Proof. We can identify $\left(2^{\mathbb{N}}\right)^{\mathbb{N}}$ with $2^{\mathbb{N} \times \mathbb{N}}$. It has the usual product topology, whose basic nbhds are given by $N_{p}=\left\{x \in 2^{\mathbb{N} \times \mathbb{N}}: x \mid(m \times n)=p\right\}$, where $p \in 2^{m \times n}$, $m, n \in \mathbb{N}$. Similarly identity $\left(2^{\mathbb{N}}\right)^{m}$ with $2^{m \times \mathbb{N}}$ with the product topology, whose basic nbhds are $N_{p}^{(m)}=\left\{x \in 2^{m \times \mathbb{N}}: x \mid(m \times n)=p\right\}$, for $p \in 2^{m \times n}, n \in \mathbb{N}$. We call $p \in 2^{m \times n}(m, n \in \mathbb{N})$ conditions.

We use below the following general notation:

$\forall^{*} x P(x)$ means " $P(x)$ holds on a comeager set",

$\forall^{+} x P(x)$ means " $P(x)$ holds on a non-meager set",

and for $U$ open,

$\forall^{*} x \in U P(x)$ means " $P$ holds on a comeager in $U$ set",

$\forall^{+} x \in U P(x)$ means " $P$ holds on a non-meager in $U$ set".

In this notation, the Kuratowski-Ulam Theorem asserts that if $P$ has the property of Baire, then

$$
\forall^{*}(x, y) P(x, y) \Leftrightarrow \forall^{*} x \forall^{*} y P(x, y) \Leftrightarrow \forall^{*} y \forall^{*} x P(x, y) .
$$

Assume now $f$ is as in the theorem. Then $f$ is Baire measurable, so $f$ is continuous on a dense $G_{\delta}$ set $G=\bigcap_{n} G_{n} \subseteq\left(2^{\mathbb{N}}\right)^{\mathbb{N}}$, where the $G_{n}$ are open, dense and decreasing.

We will construct inductively for $n \in \mathbb{N}$ :

1) conditions $p_{n}, q_{n} \in 2^{\left(l_{n} \times k_{n}\right)}$, with $l_{n}, k_{n}$ strictly increasing;

2) $x_{i}, y_{i} \in 2^{\mathbb{N}}$ for $i<l_{n}$, such that $i \mapsto x_{i}, i \mapsto y_{i}$ are injective, $x_{i} \neq y_{j}, \forall j \leq i$, $y_{i} \neq x_{j}, \forall j \leq i$ and $p_{n}=\left(x_{0}, \ldots, x_{l_{n}-1}\right)\left|k_{n}, q_{n}=\left(y_{0}, \ldots, y_{l_{n}-1}\right)\right| k_{n}$, which moreover satisfy:

(a) $N_{p_{n}}, N_{q_{n}} \subseteq G_{n}$;

(b) $\forall^{*} \alpha \in\left(2^{\mathbb{N}}\right)^{\mathbb{N}}\left(\left(x_{0}, \ldots, x_{l_{n}-1}\right) \wedge \alpha \in G \&\left(y_{0}, \ldots, y_{l_{n}-1}\right)^{\wedge} \alpha \in G\right)$;

(c) $\forall^{+} \alpha \in\left(2^{\mathbb{N}}\right)^{\mathbb{N}}\left(f\left(\left(x_{0}, \ldots, x_{l_{n}-1}\right)^{\wedge} \alpha\right)=f\left(\left(y_{0}, \ldots, y_{l_{n}-1}\right)^{\wedge} \alpha\right)\right)$.

We will write below $\bar{x}_{k}$ for $\left(x_{0}, \ldots, x_{k}\right)$ and similarly for the $y$ 's.

Assuming this can be done, by (b), (c) we can find $\left\{\alpha^{n}\right\}$ such that

$$
\bar{x}_{l_{n-1}} \widehat{\alpha^{n}}, \bar{y}_{l_{n-1}} \widehat{-} \alpha^{n} \in G
$$

and

$$
f\left(\bar{x}_{l_{n}-1} \widehat{\alpha^{n}}\right)=f\left(\bar{y}_{l_{n-1}} \widehat{\alpha}^{n}\right) .
$$

If $x=\left(x_{n}\right), y=\left(y_{n}\right)$, by (a) we have $x, y \in G$ and since $\bar{x}_{l_{n}-1} \widehat{\alpha^{n}} \rightarrow x$,

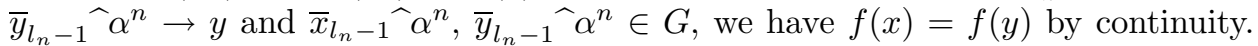
But also $n \mapsto x_{n}, n \mapsto y_{n}$ are injective and $x_{n} \neq y_{m}$ for all $n, m$, so we are done.

To show that this construction is possible, we use the following lemma:

Lemma. Let $p \in 2^{m \times n}, \varphi$ a Borel function defined on a comeager in $N_{p}$ set, such that on its domain

$$
x E_{1} y \Rightarrow \varphi(x) F \varphi(y) .
$$


Then we can find $q \in 2^{m \times n^{\prime}}$ with $q \supseteq p$ and a condition $r$ such that

$$
\forall^{*} \delta \in N_{q}^{(m)} \forall^{*} \varepsilon \in N_{q}^{(m)} \forall^{*} x \in N_{r}\left(\varphi\left(\delta^{\curlywedge} x\right)=\varphi(\widehat{\varepsilon} x)\right) .
$$

Proof. Fix $x \in\left(2^{\mathbb{N}}\right)^{\mathbb{N}}$. Define a partial function $\xi_{x}:\left(2^{\mathbb{N}}\right)^{m} \rightarrow X$ by $\xi_{x}(\delta)=\varphi\left(\delta^{\wedge} x\right)$, for $\delta \in\left(2^{\mathbb{N}}\right)^{m}$ such that $\varphi\left(\delta^{\wedge} x\right)$ is defined. By the Kuratowski-Ulam Theorem $\xi_{x}$ is defined on a comeager in $N_{p}^{(m)}$ set of $\delta$ 's, for a comeager in $\left(2^{\mathbb{N}}\right)^{\mathbb{N}}$ set of $x$ 's.

Since for $\delta, \varepsilon \in\left(2^{\mathbb{N}}\right)^{m}, \delta \prec x E_{1} \widehat{\varepsilon^{\wedge}} x$, the image of $\xi_{x}$ is contained in some $F$ equivalence class, so is countable. Thus we can find some $q_{x} \supseteq p, q_{x} \in 2^{m \times n_{x}}$, such that $\xi_{x}$ is constant on a comeager set in $N_{q_{x}}^{(m)}$. Then find conditions $r$ and $q \in 2^{m \times n^{\prime}}$ with $q \supseteq p$ such that on a comeager in $N_{r}$ set of $x$ 's, $q_{x}=q$. Then we have

$$
\forall^{*} x \in N_{r} \forall^{*} \delta \in N_{q}^{(m)} \forall^{*} \varepsilon \in N_{q}^{(m)}\left(\varphi\left(\delta^{\prec} x\right)=\varphi(\widehat{\varepsilon} x)\right),
$$

and so, by Kuratowski-Ulam,

$$
\forall^{*} \delta \in N_{1}^{(m)} \forall^{*} \varepsilon \in N_{q}^{(m)} \forall^{*} x \in N_{r}\left(\varphi\left(\delta^{\prec} x\right)=\varphi\left(\varepsilon^{\prec} x\right)\right) .
$$

We now construct the $p_{n}, q_{n}, x_{n}, y_{n}$. Assume the construction has been completed up to $n$. By (b), (c), find a condition $p^{\prime}$ such that

$$
\forall^{*} \alpha \in N_{p^{\prime}}\left[f\left(\bar{x}_{l_{n}-1} \widehat{\alpha}\right)=f\left(\bar{y}_{l_{n}-1} \widehat{\alpha}\right) \& \bar{x}_{l_{n}-1} \widehat{\alpha}, \bar{y}_{l_{n}-1} \widehat{\alpha} \in G\right] .
$$

Fix such an $\alpha$. As $\bar{x}_{l_{n}-1} \widehat{\alpha}, \bar{y}_{l_{n}-1} \widehat{\alpha} \in G$, we can find conditions $p_{n+1}^{\prime}, q_{n+1}^{\prime} \in$ $2^{l_{n+1}^{\prime} \times k_{n+1}^{\prime}}$ with $l_{n+1}^{\prime}>l_{n}, k_{n+1}^{\prime}>k_{n}$ and $p_{n+1}^{\prime} \supseteq p_{n} \sqcup p^{\prime}, q_{n+1}^{\prime} \supseteq q_{n} \sqcup p^{\prime}$ (where $p_{n} \sqcup p^{\prime} \mid\left(l_{n} \times k_{n}\right)=p_{n}$ and $p_{n} \sqcup p^{\prime}\left(l_{n}+i, j\right)=p^{\prime}(i, j)$ and similarly for $\left.q_{n} \sqcup p^{\prime}\right)$, $p_{n+1}^{\prime} \subseteq \bar{x}_{l_{n-1}} \widehat{\alpha} \alpha, q_{n+1}^{\prime} \subseteq \bar{y}_{l_{n-1}} \widehat{\alpha} \alpha, N_{p_{n+1}^{\prime}}, N_{q_{n+1}^{\prime}} \subseteq G_{n+1}$. Notice that

$$
p_{n+1}^{\prime}\left|\left(\left[l_{n}, l_{n+1}^{\prime}\right) \times k_{n+1}^{\prime}\right)=q_{n+1}^{\prime}\right|\left(\left[l_{n}, l_{n+1}^{\prime}\right) \times k_{n+1}^{\prime}\right)=\alpha \mid\left(\left[0, l_{n+1}^{\prime}-l_{n}\right) \times k_{n+1}^{\prime}\right) .
$$

Define $p \in 2^{\left(l_{n+1}^{\prime}-l_{n}\right) \times k_{n+1}^{\prime}}$ by $p(i, j)=p_{n+1}^{\prime}\left(l_{n}+i, j\right)$. Thus $p \supseteq p^{\prime}$.

Use the lemma for this $p$ and $\varphi(x)=f\left(\bar{x}_{l_{n}-1} \widehat{x} x\right)=f\left(\bar{y}_{l_{n}-1} \widehat{x}\right)$, to obtain $q$ and $r$ as in the lemma.

We have then, if $m=l_{n+1}^{\prime}-l_{n}$ :

$$
\begin{aligned}
& \forall^{*} \delta \in N_{q}^{(m)} \forall^{*} \varepsilon \in N_{q}^{(m)} \forall^{*} x \in N_{r} \\
& {\left[f\left(\bar{x}_{l_{n}-1} \widehat{\varepsilon} x\right)=f\left(\bar{y}_{l_{n}-1} \widehat{\delta} \widehat{\chi} x\right)=f\left(\bar{x}_{l_{n}-1} \widehat{\delta} \widehat{\chi} x\right)=f\left(\bar{y}_{l_{n}-1} \widehat{\varepsilon} x\right)\right],}
\end{aligned}
$$

and by (b)

$$
\begin{aligned}
& \forall^{*} \delta \in N_{q}^{(m)} \forall^{*} \varepsilon \in N_{q}^{(m)} \forall^{*} x \in\left(2^{\mathbb{N}}\right)^{\mathbb{N}} \\
& \left(\bar{x}_{l_{n}-1} \widehat{\widehat{\varepsilon} x} x \in G \& \bar{x}_{l_{n}-1} \widehat{\widehat{\delta}} x \in G\right. \\
& \left.\& \bar{y}_{l_{n-1}} \widehat{\varepsilon} \widehat{\varepsilon} x \in G \& \bar{y}_{l_{n}-1} \widehat{\delta} \widehat{\delta} x \in G\right) \text {. }
\end{aligned}
$$


Since the set of all $(\delta, \varepsilon) \in N_{q}^{(m)} \times N_{q}^{(m)}$ which satisfy at least one of the following conditions:

$$
\begin{array}{ll}
\delta_{i}=x_{j} & \text { for some } i<m, j<l_{n}, \\
\delta_{i}=\delta_{j} & \text { for some } j \neq i<m, \\
\varepsilon_{i}=y_{j} & \text { for some } i<m, j<l_{n}, \\
\varepsilon_{i}=\varepsilon_{j} & \text { for some } j \neq i<m, \\
\delta_{i}=\varepsilon_{j} & \text { for some } j \leq i<m, \\
\varepsilon_{i}=\delta_{j} & \text { for some } j \leq i<m, \\
\delta_{i}=y_{j} & \text { for some } i<m, j<l_{n}, \\
\varepsilon_{i}=x_{j} & \text { for some } i<m, j<l_{n},
\end{array}
$$

is meager in $N_{q}^{(m)} \times N_{q}^{(m)}$, we can find $x_{l_{n}}, \ldots, x_{l_{n}+m-1}, y_{l_{n}}, \ldots, y_{l_{n}+m-1}$ with $i \mapsto x_{i}, i \mapsto y_{i}$ injective for $i<l_{n}+m$ and $x_{i} \neq y_{j}, y_{i} \neq x_{j}$ for $j \leq i<l_{n}+m$ and such that for $l_{n+1}=l_{n}+m$

$$
\forall^{*} \alpha \in N_{r}\left[f\left(\bar{x}_{l_{n+1}-1} \widehat{\alpha}\right)=f\left(\bar{y}_{l_{n+1}-1} \widehat{\alpha}\right)\right]
$$

and

$$
\forall^{*} \alpha \in\left(2^{\mathbb{N}}\right)^{\mathbb{N}}\left[\bar{x}_{l_{n+1}-1} \widehat{\alpha} \in G \& \bar{y}_{l_{n+1}-1} \widehat{\alpha} \in \in\right] .
$$

Finally, choose $k_{n+1}$ large enough.

\section{THE MAIN THEOREM}

Our main result is that up to Borel bireducibility, $E_{0}$ and $E_{1}$ are the only nonsmooth Borel hypersmooth equivalence relations. More precisely we have the following:

Theorem 2.1. Let $E$ be a hypersmooth Borel equivalence relation. Then exactly one of the following holds:

(I) $E \leq E_{0}$;

(II) $E_{1} \sqsubseteq E$.

Since by [HKL], if $E$ is non-smooth Borel, then $E_{0} \sqsubseteq E$, it follows that for any hypersmooth Borel $E$, exactly one of the following holds:

(i) $E$ is smooth;

(ii) $E \approx^{*} E_{0}$;

(iii) $E \approx E_{1}$.

The proof of 2.1 uses the methods of effective descriptive set theory. In fact we prove the following effective result.

Theorem 2.2. Let $\left\{F_{n}\right\}$ be a sequence of equivalence relations on the Baire space $\mathcal{N}$ such that $F_{0} \subseteq F_{1} \subseteq \cdots$ and each $F_{n}$ is $\Pi_{1}^{0}$, uniformly on $n$. Let $E=\bigcup_{n} F_{n}$. Then exactly one of the following holds:

(I) $E \leq E_{0}$ via a $\Delta_{1}^{1}$ map;

(II) $E_{1} \sqsubseteq E$ via a continuous embedding.

Before we prove 2.2, let us argue that it, and its obvious relativization, implies 2.1. Indeed by the relativized version of 2.2 , if $F_{0} \subseteq F_{1} \subseteq \cdots$ is an increasing sequence of closed equivalence relations in $\mathcal{N}$ and $E=\bigcup_{n} F_{n}$, then either $E \leq$ $E_{0}$ or $E_{1} \sqsubseteq E$ (via a continuous function). Assume now that $E$ is an arbitrary hypersmooth Borel equivalence relation on the standard Borel space $X$. Then (see 
$[K 3,13.11])$ there is a Polish topology on $X$ generating its Borel structure and closed relations in this topology $F_{0} \subseteq F_{1} \subseteq \cdots$, with $E=\bigcup_{n} F_{n}$. Let $C \subseteq \mathcal{N}$ be closed and $\pi: C \rightarrow X$ a continuous injective map from $C$ onto $X$.

Define $F_{n}^{\prime}$ on $\mathcal{N}$ by

$$
x F_{n}^{\prime} y \Leftrightarrow(x=y) \text { or }\left[x, y \in C \& \pi(x) F_{n} \pi(y)\right] .
$$

Then $F_{0}^{\prime} \subseteq F_{1}^{\prime} \subseteq \cdots$ and each $F_{n}^{\prime}$ is a closed equivalence relation on $\mathcal{N}$. Let $E^{\prime}=\bigcup_{n} F_{n}^{\prime}$. Then $E^{\prime} \leq E_{0}$ or $E_{1} \sqsubseteq E^{\prime}$ (via a continuous function). If $E^{\prime} \leq E_{0}$ via $f$, then $E \leq E_{0}$ via $f \circ \pi^{-1}$. If $E_{1} \sqsubseteq E^{\prime}$ via a continuous embedding $g$, then $g\left[\left(2^{\mathbb{N}}\right)^{\mathbb{N}}\right] \subseteq C$, so $\pi \circ g$ is a continuous embedding of $\left(2^{\mathbb{N}}\right)^{\mathbb{N}}$ into $X$, which witnesses that $E_{1} \sqsubseteq E$.

Proof of 2.2. For each $n<m$, put

$$
Y_{n, m}=\bigcup\left\{A \in \Sigma_{1}^{1}: A^{2} \cap F_{m} \subseteq F_{n}\right\} .
$$

By the First Reflection Theorem, if $A \in \Sigma_{1}^{1}$ and $A^{2} \cap F_{m} \subseteq F_{n}$, there is $B \in \Delta_{1}^{1}$, $B \supseteq A$ with $B^{2} \cap F_{m} \subseteq F_{n}$, so

$$
Y_{n, m}=\bigcup\left\{A \in \Delta_{1}^{1}: A^{2} \cap F_{m} \subseteq F_{n}\right\}
$$

and in particular $Y_{n, m}$ is $\Pi_{1}^{1}$, uniformly in $n, m$. Put

$$
X_{n, m}=\mathcal{N} \backslash Y_{n, m}, \quad X^{*}=\bigcap_{n} \bigcup_{m>n} X_{n, m}
$$

Thus $X^{*} \in \Sigma_{1}^{1}$.

Case I. $X^{*}=\varnothing$.

We show then that (I) holds. Since $\mathcal{N}=\bigcup_{n} \bigcap_{m>n} Y_{n, m}$, by effective reduction we can find a pairwise disjoint sequence $\left\{S_{n}\right\}$ of $\Delta_{1}^{1}$ sets, uniformly in $n$, such that $\bigcup_{n} S_{n}=\mathcal{N}$ and $S_{n} \subseteq \bigcap_{m>n} Y_{n, m}$, i.e.,

$$
\forall x \in S_{n} \forall m>n \exists A \in \Delta_{1}^{1}\left(x \in A \& A^{2} \cap F_{m} \subseteq F_{n}\right) .
$$

For equivalence relations $R \subseteq S$, we say that $S / R$ is countable, if every $S$ equivalence class contains only countably many $R$-equivalence classes. We claim now that $\left(E \mid S_{n}\right) /\left(F_{n} \mid S_{n}\right)$ is countable: It is clearly enough to show that $\left(F_{m} \mid S_{n}\right) /\left(F_{n} \mid S_{n}\right)$ is countable for any $m>n$. But if $C$ is an $F_{m} \mid S_{n}$-equivalence class and $D \subseteq C$ an $F_{n} \mid S_{n}$-equivalence class, then there is a $\Delta_{1}^{1}$ nonempty set $A$ such that $A \cap C \subseteq D$, so clearly there are only countably many such $D$ in $C$.

Now define a new equivalence relation $F_{0}^{\prime}$ on $\mathcal{N}$ by

$$
x F_{0}^{\prime} y \Leftrightarrow \exists n\left(x, y \in S_{n} \& x F_{n} y\right) .
$$

Clearly $F_{0}^{\prime} \subseteq E, F_{0}^{\prime}$ is $\Delta_{1}^{1}$ and smooth. Moreover, $E / F_{0}^{\prime}$ is countable. Put also, for $n>0$,

$$
\begin{aligned}
x F_{n}^{\prime} y \Leftrightarrow & \left(x, y \in \bigcup_{n^{\prime} \leq n} S_{n^{\prime}} \& x F_{n} y\right) \text { or } \\
& \exists m>n\left(x, y \in S_{m} \& x F_{m} y\right) .
\end{aligned}
$$

Then $F_{n}^{\prime}$ is smooth $\Delta_{1}^{1}$, uniformly on $n, F_{0}^{\prime} \subseteq F_{1}^{\prime} \subseteq \cdots$ and $E=\bigcup_{n} F_{n}^{\prime}$.

Now let $\varphi: \mathcal{N} \rightarrow 2^{\mathbb{N}}$ be $\Delta_{1}^{1}$ such that

$$
x F_{0}^{\prime} y \Leftrightarrow \varphi(x)=\varphi(y) .
$$

Put $\varphi[\mathcal{N}]=A \subseteq 2^{\mathbb{N}}$, so that $A \in \Sigma_{1}^{1}$. 
Let $\left\{C_{k}^{(n)}\right\}_{n, k \in \mathbb{N}}$ be a uniformly $\Delta_{1}^{1}$ family of sets such that for each $n,\left\{C_{k}^{(n)}\right\}_{k \in \mathbb{N}}$ is a separating family for $F_{n}^{\prime}$. Define the equivalence relation $\bar{F}_{n}$ on $A$ and subsets $\bar{C}_{k}^{(n)}$ of $A$ by

$$
\begin{aligned}
& \alpha \bar{F}_{n} \beta \Leftrightarrow \exists x \exists y\left[\varphi(x)=\alpha \& \varphi(y)=\beta \& x F_{n}^{\prime} y\right], \\
& \alpha \in \bar{C}_{k}^{(n)} \Leftrightarrow \exists x\left[\varphi(x)=\alpha \& x \in C_{k}^{(n)}\right] .
\end{aligned}
$$

Since $F_{0}^{\prime} \subseteq F_{n}^{\prime}$ and each $C_{k}^{(n)}$ is $F_{n}^{\prime}$-invariant, we also have for $\alpha, \beta \in A$ :

$$
\begin{aligned}
& \alpha \bar{F}_{n} \beta \Leftrightarrow \forall x \forall y\left[\varphi(x)=\alpha \& \varphi(y)=\beta \Rightarrow x F_{n}^{\prime} y\right], \\
& \alpha \in \bar{C}_{k}^{(n)} \Leftrightarrow \forall x\left[\varphi(x)=\alpha \Rightarrow x \in C_{k}^{(n)}\right] .
\end{aligned}
$$

Thus $\bar{F}_{n},\left\{\bar{C}_{k}^{(n)}\right\}$ are uniformly $\Delta_{1}^{1}$ on $A$. Clearly $\left\{\bar{C}_{k}^{(n)}\right\}$ is a separating family for $\bar{F}_{n}$. Also if $\bar{E}=\bigcup_{n} \bar{F}_{n}$, then $\bar{E}$ is a countable $\Delta_{1}^{1}$ equivalence relation on $A$.

Now let $\left\{\overline{\bar{C}}_{k}^{(n)}\right\}$ be uniformly $\Delta_{1}^{1}$ such that $\bar{C}_{k}^{(n)}=A \cap \overline{\bar{C}}_{k}^{(n)}$. Consider then the statements (1)-(6) below, in variables $\widetilde{A} \subseteq 2^{\mathbb{N}}$ and $\widetilde{F}=\left\{\widetilde{F}_{n}\right\}_{n \in \mathbb{N}}, \widetilde{F} \subseteq \mathbb{N} \times 2^{\mathbb{N}} \times 2^{\mathbb{N}}$ :

(1) $\widetilde{F}_{n}$ is an equivalence relation on $\widetilde{A}$;

(2) $\forall x \in \widetilde{A} \forall y \in \widetilde{A}\left(x \widetilde{F}_{n} y \Rightarrow y \in \Delta_{1}^{1}(x)\right)$;

(3) $\widetilde{F}_{n} \subseteq \widetilde{F}_{n+1}$;

(4) $\forall n \forall k\left[\overline{\bar{C}}_{k}^{(n)} \cap \widetilde{A}\right.$ is $\widetilde{F}_{n}$-invariant $]$;

(5) $\forall x \forall y\left[x \in \widetilde{A} \& y \in \widetilde{A} \& \neg x \widetilde{F}_{n} y \Rightarrow \exists k\left(x \in \overline{\bar{C}}_{k}^{(n)} \& y \notin \overline{\bar{C}}_{k}^{(n)}\right)\right]$;

(6) $\forall x \forall y\left[x \widetilde{F}_{n} y \& x \in \widetilde{A} \& y \in \widetilde{A} \Rightarrow x \bar{F}_{n} y\right]$.

These are clearly satisfied by $A$ and $\bar{F}=\left\{\bar{F}_{n}\right\}_{n \in \mathbb{N}}$. They also have the form for applying the Burgess Reflection Theorem. (It is understood here that in (6) we use a $\Pi_{1}^{1}$ definition for $\bar{F}_{n}$.) So we can find $\Delta_{1}^{1}$ sets $A^{*} \supseteq A, F^{*}=\left\{F_{n}\right\}, F_{n}^{*} \supseteq \bar{F}_{n}$, still satisfying (1)-(6). By (1) and (3), $F_{n}^{*}$ are increasing equivalence relations on $A^{*}$, while each $F_{n}^{*}$ is countable by (2), thus so is $E^{*}=\bigcup_{n} F_{n}^{*}$. Also (4), (5) imply that $\left\{\overline{\bar{C}}_{k}^{(n)} \cap A\right\}_{k \in \mathbb{N}}$ is a separating family for $F_{n}^{*}$, so $F_{n}^{*}$ is smooth. Finally, (6) shows that $E^{*} \mid A=\bar{E}$.

Since $E^{*}$ is hypersmooth and countable, by [DJK] $E^{*}$ can be reduced by a $\Delta_{1}^{1}$ function to $E_{0}$. Since $\varphi$ reduces $E$ to $E^{*}$ as well, we have that $\psi \circ \varphi$ is a $\Delta_{1}^{1}$ reduction of $E$ to $E_{0}$.

This completes Case I.

Case II. $X^{*} \neq \varnothing$.

We will show then that (II) holds. Since $X^{*}$ is nonempty $\Sigma_{1}^{1}$, the set

$$
X=X^{*} \cap\left\{x \in \mathcal{N}: \omega_{1}^{x}=\omega_{1}^{\mathrm{CK}}\right\}
$$

is also $\Sigma_{1}^{1}$ and nonempty.

Note that the Gandy-Harrington topology when restricted to $\left\{x \in \mathcal{N}: \omega_{1}^{x}=\right.$ $\left.\omega_{1}^{\mathrm{CK}}\right\}$ has a clopen basis (since the intersection of a $\Pi_{1}^{1}$ set with this set is a countable union of $\Delta_{1}^{1}$ sets), so regular; thus by the Choquet Criterion (see, e.g., [K3, 8.18]) it is Polish. Denote the Gandy-Harrington topology restricted to $X$ by $\tau$. Fix also a complete metric $d$ for $\tau$ on $X$. We can of course assume that $d \geq \delta$, where $\delta$ is the ordinary metric on $\mathcal{N}$. We will embed $E_{1}$ into $E \mid X$ (continuously for the ordinary topology on $\mathcal{N}$.) 
Fix the canonical bijection \langle\rangle of $\mathbb{N}^{2}$ with $\mathbb{N}$ given by the Cantor diagonal enumeration, i.e.,

$$
\langle n, k\rangle=\frac{(n+k)(n+k+1)}{2}+k .
$$

For $s \in 2^{p}$, where $p=\langle n, k\rangle$, and $j \in \mathbb{N}$ we let $s_{j}(i)=s(\langle j, i\rangle)$, provided $\langle j, i\rangle<p$. This associates to $s$ a sequence $\left\langle s_{j}: j \in \mathbb{N}\right\rangle$ of finite sequences, which are eventually $\varnothing$. Put

$$
L(p)=\min \left\{j: s_{j}=\varnothing\right\}=\min \{j:\langle j, 0\rangle \geq p\} .
$$

Define also for $s, t \in 2^{p}, j \leq L(p)$,

$$
s \sim_{j} t \Leftrightarrow \forall j^{\prime} \geq j\left(s_{j^{\prime}}=t_{j^{\prime}}\right) .
$$

Then $\sim_{j}$ is an equivalence relation on $2^{p}$ and $\sim_{0} \subseteq \sim_{1} \subseteq \cdots \subseteq \sim_{L(p)}$. Moreover, $\sim_{0}$ is equality and $\sim_{L(p)}=2^{p} \times 2^{p}$.

For $\alpha \in 2^{\mathbb{N}}$, let also $\alpha_{m}(k)=\alpha(\langle m, k\rangle)$. Then, identifying $\alpha \in 2^{\mathbb{N}}$ with $\left\{\alpha_{m}\right\}_{m \in \mathbb{N}} \in\left(2^{\mathbb{N}}\right)^{\mathbb{N}}$, we have that

$$
\begin{aligned}
\alpha E_{1} \beta & \Leftrightarrow \exists n \forall m \geq n\left(\alpha_{m}=\beta_{m}\right) \\
& \Leftrightarrow \exists n \forall p\left(n \leq L(p) \Rightarrow \alpha\left|p \sim_{n} \beta\right| p\right) .
\end{aligned}
$$

For an equivalence relation $E$ (on some set $S$ ) and sets $A, B(\subseteq S$ ) let

$$
A E B \Leftrightarrow \forall x \in A \exists y \in B(x E y) \& \forall y \in B \exists x \in A(x E y) .
$$

Note that $A E B \Leftrightarrow[A]_{E}=[B]_{E}$, and this is an equivalence relation too.

We first claim that in order to embed $E_{1}$ into $E \mid X$ (continuously), it is enough to build a family $\left\{U_{s}\right\}_{s \in 2<\mathbb{N}}$ and a strictly increasing function $N: \mathbb{N} \rightarrow \mathbb{N}$ satisfying:

(i) $U_{s}$ is a nonempty $\Sigma_{1}^{1}$ subset of $X, t \supsetneqq s \rightarrow \bar{U}_{t}^{\tau} \subset U_{s}, U_{s \wedge 0} \cap U_{s \wedge 1}=\varnothing$ and $d\left(U_{s \wedge i}\right) \leq 2^{-l h(s)}$.

(ii) If $s, t \in 2^{p}, p=\langle n, k\rangle$ and $s \sim_{n} t$, then $\left(U_{s^{\wedge} 0} \times U_{t^{\wedge} 1}\right) \cap F_{n+1}=\varnothing$.

(iii) If $s, t \in 2^{p}, p=\langle n, k\rangle, j \leq L(p)$ and $s \sim_{j} t$, then $U_{s} F_{N(j)} U_{t}$.

Indeed, assume this can be done. For $\alpha \in 2^{\mathbb{N}}$, define $f(\alpha)$ by $\{f(\alpha)\}=\bigcap_{n} U_{\alpha \mid n}$. This is clearly well-defined and 1-1 by (i). It is also continuous for the ordinary topology on $\mathcal{N}$ as $d \geq \delta$. We argue that $f$ embeds $E_{1}$ into $E$.

Suppose that $\alpha E_{1} \beta$, say $\alpha_{m}=\beta_{m}$ for $m \geq n$. Then for $p$ such that $n \leq L(p)$, $\alpha\left|p \sim_{n} \beta\right| p$. By (iii) $U_{\alpha \mid p} F_{N(n)} U_{\beta \mid p}$, so there are $\alpha_{p} \in U_{\alpha \mid p}, \beta_{p} \in U_{\beta \mid p}$ with $\alpha_{p} F_{N(n)} \beta_{p}$. Since $\alpha_{p} \rightarrow f(\alpha), \beta_{p} \rightarrow f(\beta)$ in the ordinary topology on $\mathcal{N}$ and $F_{N(n)}$ is closed in that topology, $f(\alpha) F_{N(n)} f(\beta)$, so $f(\alpha) E f(\beta)$.

Assume now $(\alpha, \beta) \notin E_{1}$. Let $p=\langle n, k\rangle$ be such that $\alpha(p) \neq \beta(p)$. Let $n_{0}$ be smallest with $\alpha\left|(p+1) \sim_{n_{0}+1} \beta\right|(p+1)$. Clearly $n_{0} \geq n$. Let $k_{0}$ be least with $\alpha\left(p_{0}\right) \neq \beta\left(p_{0}\right)$ for $p_{0}=\left\langle n_{0}, k_{0}\right\rangle$. Thus $p_{0} \leq p$. Now $\alpha\left|p_{0} \sim_{n_{0}} \beta\right| p_{0}$, so by (ii) $\left(U_{\alpha \mid\left(p_{0}+1\right)} \times U_{\beta \mid\left(p_{0}+1\right)}\right) \cap F_{n_{0}+1}=\varnothing$ and thus $\left(U_{\alpha \mid\left(p_{0}+1\right)} \times U_{\beta \mid\left(p_{0}+1\right)}\right) \cap F_{n}=\varnothing$, so $\neg f(\alpha) F_{n} f(\beta)$. Since this happens for infinitely many $n, \neg f(\alpha) E f(\beta)$.

For the rest of the proof, let us introduce the following terminology:

Given $n<m$ and $\varnothing \neq A \in \Sigma_{1}^{1}, A \subseteq X$, we will say that $F_{n}$ is meager in $F_{m}$ on $A$ if $F_{n}$ is meager in $F_{m}$ on $A^{2}$ with the $\tau \times \tau$-topology.

Since $F_{n}, F_{m}$ are both closed in the product of the ordinary topology and thus in the $(\tau \times \tau)$-topology, this means that there are no nonempty $\Sigma_{1}^{1}$ subsets $C, D \subseteq A$ with $\varnothing \neq(C \times D) \cap F_{m} \subseteq F_{n}$. We claim that this is equivalent to saying that there is no nonempty $\Sigma_{1}^{1}$ set $B \subseteq A$ with $B^{2} \cap F_{m} \subseteq F_{n}$. Indeed, if such $C, D$ exist, we can assume first that $C F_{m} D$ by replacing them by $C \cap[D]_{F_{m}}, D \cap[C]_{F_{m}}$. We claim 
then that $C^{2} \cap F_{m} \subseteq F_{n}$. Indeed, if $x, y \in C$ and $x F_{m} y$, find $z \in D$ with $x F_{m} z$, hence $x F_{n} z$. Also $y F_{m} z$, hence $y F_{n} z$ and thus $x F_{n} y$.

We can use this argument to prove immediately the following basic lemma.

Lemma 2.3. Let $A \subseteq X$ be a nonempty $\Sigma_{1}^{1}$ set and $x_{1}, \ldots, x_{k} \in A$. For any $n \in \mathbb{N}$ there is $m>n$ and $a \Sigma_{1}^{1}$ set $A^{*} \subseteq A$ such that $x_{1}, \ldots, x_{k} \in A^{*}$ and $F_{n}$ is meager in $F_{m}$ on $A^{*}$.

Proof. Recall that $X \subseteq X^{*}=\bigcap_{n} \bigcup_{m>n} X_{m}$. So $A \subseteq \bigcup_{m>n} X_{m, n}$. So find $m>n$ with $x_{1}, \ldots, x_{k} \in X_{m, n}$. This can be done as $\left\{X_{n, m}\right\}_{m>n}$ is increasing. Put $A^{*}=A \cap X_{n, m}$. If $F_{n}$ is not meager in $F_{m}$ on $A^{*}$, then by the preceding argument, there is a nonempty $\Sigma_{1}^{1}$ set $B \subseteq A^{*}$ with $B^{2} \cap F_{m} \subseteq F_{n}$, so by the definition of $Y_{n, m}, B \subseteq Y_{n, m}$, so $B=\varnothing$, a contradiction.

In order to construct the family $\left\{U_{s}\right\}$ and the function $N$ satisfying (i)-(iii) above, we will impose the following requirements:

- $R(0): U_{\varnothing}$ will be a nonempty $\Sigma_{1}^{1}$ subset of $X$ and $N(0)>0$ will be such that $F_{0}$ is meager in $F_{N(0)}$ on $U_{\varnothing}$.

- $R(1)$ (as (i) before): For $s \in 2^{p}, i=0$ or $1, \bar{U}_{s^{\wedge} i}^{\tau} \subseteq U_{s}, U_{s^{\wedge} 0} \cap U_{s^{\wedge} 1}=\varnothing$ and $d\left(U_{s^{\wedge} i}\right) \leq 2^{-p}$

- $R(2)$ (as (ii) before): For $s, t \in 2^{p}, p=\langle n, k\rangle$, if $s \sim_{n} t$, then $\left(U_{s^{\wedge} 0} \times U_{t^{\wedge} 1}\right) \cap$ $F_{n+1}=\varnothing$.

- $R(3)$ : For $j \leq L(p+1), F_{j}$ is meager in $F_{N(j)}$ on $\bigcup_{s \in 2^{p+1}} U_{s}$.

- $R(4)$ : (a) For $s, t \in 2^{p}, j \leq L(p), i=0$ or 1 :

$$
s \sim_{j} t \Rightarrow U_{s^{\wedge} i} F_{N(j)} U_{t ` i}
$$

(b) for $s \in 2^{p}, p=\langle n, k\rangle$,

$$
U_{s\urcorner 0} F_{N(n+1)} U_{s\urcorner 1} \text {. }
$$

We claim that these are enough, i.e., they imply (i)-(iii).

Clearly $R(0)-(2) \Rightarrow(\mathrm{i})$, (ii). We will verify that $R(4) \Rightarrow$ (iii):

Assume $R(4)$. We have to show that for all $p, s, t \in 2^{p}, j \leq L(p)$, if $s \sim_{j} t$, then $U_{s} F_{N(j)} U_{t}$. This is clear for $p=0$. Suppose it holds for $p=\langle n, k\rangle$ and consider $p+1, s, t \in 2^{p+1}$, say $s=\bar{s} \wedge i, t=\bar{t}^{\wedge} i^{\prime}$ with $\bar{s}, \bar{t} \in 2^{p}, j \leq L(p+1)$. First let $i=i^{\prime}$ : If $j \leq L(p)$, then $\bar{s} \hat{i} \sim_{j} \bar{t} \hat{i}$ implies $\bar{s} \sim_{j} \bar{t}$, so by $\bar{R}(4)(\mathrm{a}) U_{s} F_{N(j)} U_{t}$. If $j=L(p+1)$, since $\bar{s} \sim_{L(p)} \bar{t}$, by $R(4)\left(\right.$ a) again we have $U_{s} F_{N(L(p))} U_{t}$, so $U_{s} F_{N(j)} U_{t}$, since $L(p+1) \geq L(p)$ and $N$ is increasing. Consider now the case $i \neq i^{\prime}$, say $i=0, i^{\prime}=1$. If $\left.\bar{s}\right\urcorner 0 \sim_{j} \bar{t} 1$, then $\bar{s} \wedge 1 \sim_{j} \bar{t} 1$, so, by the first case $\left(i=i^{\prime}\right)$, we have $U_{\bar{s}{ }^{\wedge}} F_{N(j)} U_{\bar{t} \wedge 1}$. By $R(4)$ (b) also, $U_{\bar{s}{ }^{\wedge} 0} F_{N(n+1)} U_{\bar{s} \wedge 1}$. But $\bar{s}{ }^{\wedge}, \bar{t}{ }^{\wedge} 1$ differ at $p=\langle n, k\rangle$, so clearly $j \geq n+1$ and, since $N$ is increasing, $N(j) \geq N(n+1)$, so $U_{\bar{s} \wedge 0} F_{N(j)} U_{\bar{s} \wedge 1}$. By transitivity $U_{\bar{s}^{\wedge} 0} F_{N(j)} U_{\bar{t}^{\wedge} 1}$, and we are done.

We construct now, by induction on $p \in \mathbb{N},\left\{U_{s}\right\}_{s \in 2^{p}}$ and $N(j)$, for $j \leq L(p)$, satisfying $R(0)-(4)$.

For $p=0$, we choose a nonempty $\Sigma_{1}^{1}$ set $U_{\varnothing} \subseteq X$ and $N(0)>0$, so that $F_{0}$ is meager in $F_{N(0)}$ on $U_{\varnothing}$. This can be done by Lemma 2.3 .

For the inductive step we will need some new concepts and a few combinatorial lemmas.

A tree is a finite undirected graph which is connected and has no loops. A labelled tree is a tree $T$ together with an assignment $(s, t) \mapsto n(s, t)$ which gives 
for each edge $(s, t)$ of $T$ a natural number $n(s, t)$ (its label). We usually write $s \frac{n}{-} t$ if $n(s, t)=n$.

By a tree structure we mean a triple $(T, U, M)$, where

(i) $T$ is a labelled tree;

(ii) $U$ is a map assigning to each vertex $s$ of $T$ a nonempty $\Sigma_{1}^{1}$ set $U(s)=U_{s} \subseteq X$;

(iii) $M$ is a mapping from the set of labels of $T$ into $\mathbb{N}$.

A tree structure $(T, U, M)$ is good if moreover

(iv) $s \stackrel{n}{-} t \Rightarrow U_{s} F_{M(n)} U_{t}$.

A tree structure $\left(T, U^{\prime}, M\right)$ refines $(T, U, M)$ if $U_{s}^{\prime} \subseteq U_{s}$ for every vertex $s$ of $T$.

Lemma 2.4. (i) Let $U, V$ be $\Sigma_{1}^{1}$ nonempty sets and $x \in U, y \in V$. If $F$ is a $\Sigma_{1}^{1}$ equivalence relation and $x F y$, then there are nonempty $\Sigma_{1}^{1}$ sets $U^{\prime} \subseteq U, V \subseteq V$ with $x \in U^{\prime}, y \in V^{\prime}$ and $U^{\prime} F V^{\prime}$.

(ii) If $U, V$ are nonempty $\Sigma_{1}^{1}$ sets, $F$ a $\Sigma_{1}^{1}$ equivalence relation and $U F V$, then for any nonempty $\Sigma_{1}^{1}$ set $A \subseteq U$, we can find a nonempty $\Sigma_{1}^{1}$ set $B \subseteq V$ with $A F B$. Moreover, if $x \in A, y \in V$ and $x F y$ then $y \in B$.

Proof. (i) Let $U^{\prime}=U \cap[V]_{F}$ and $V^{\prime}=V \cap[U]_{F}$.

(ii) Let $B=V \cap[A]_{F}$.

Lemma 2.5. Let $(T, U, M)$ be a good tree structure. Let $s_{0}$ be a vertex of $T$ and $A$ a nonempty $\Sigma_{1}^{1}$ subset of $U_{s_{0}}$. Then there is a refinement $\left(T, U^{\prime}, M\right)$ of $(T, U, M)$ which is good, such that $U_{s_{0}}^{\prime}=A$. Moreover, if $x_{s} \in U_{s}$ for all vertices and $s \stackrel{n}{-} t \Rightarrow x_{s} F_{M(n)} x_{t}$, then if $x_{s_{0}} \in A$ we can insure that $x_{s} \in U_{s}^{\prime}$, for all $s$.

Proof. Let $l(s, t)$ be the distance function of $T$, i.e., the length of the unique path from $s$ to $t$. Let $l(s)=l\left(s, s_{0}\right)$. We will define $U_{s}^{\prime}$ by induction on $l(s)$. For $l(s)=0$, i.e., $s=s_{0}$, we have $U_{s_{0}}^{\prime}=A$. Assume now $l(s)>0$ and let $t$ be the vertex following $s$ on the unique path from $s$ to $s_{0}$, so that $l(t)=l(s)-1$. Thus $U_{t}^{\prime}$ has been defined. If $s \frac{n}{-} t$, then $U_{s} F_{M(n)} U_{t}$ and, since $U_{t}^{\prime} \subseteq U_{t}$, we can find $U_{s}^{\prime} \subseteq U_{s}$ so that $U_{s}^{\prime} F_{M(n)} U_{t}^{\prime}$, by $2.4(\mathrm{ii})$.

Lemma 2.6. Let $(T, U, M)$ be a tree structure and $x_{s} \in U_{s}$ for every vertex $s$ of T. If

$$
s \stackrel{n}{-} t \Rightarrow x_{s} F_{M(n)} x_{t}
$$

then there is a refinement $\left(T, U^{\prime}, M\right)$ of $(T, U, M)$ which is good and $x_{s} \in U_{s}^{\prime}$ for all $s$.

Proof. By induction on the cardinality of the set of vertices of $T$. Let $s$ be a terminal vertex of $T$, i.e., one which belongs to a unique edge. Let $t$ be the other vertex of this edge. If we delete $s$ and this unique edge (but not $t$ ), we obtain a new tree $T^{*}$ with one fewer vertex. Let $\left(U^{*}, M^{*}\right)$ be $(U, M) \mid T^{*}$. By the induction hypothesis, there is a good refinement $\left(T^{*}, U^{*^{\prime}}, M^{*}\right)$ of $\left(T^{*}, U^{*}, M^{*}\right)$ satisfying $x_{s^{*}} \in U_{s^{*}}^{*^{\prime}}$ for $s^{*} \neq s$. Applying 2.4(i) to $F_{M(n)}$, where $s \stackrel{n}{-} t, U_{s}, U_{t}^{*^{\prime}}$ and the points $x_{s}, x_{t}$, we can find $U_{s}^{\prime} \subseteq U_{s}, U_{t}^{\prime} \subseteq U_{t}^{*^{\prime}}$ with $x_{s} \in U_{s}^{\prime}, x_{t} \in U_{t}^{\prime}$ and $U_{s}^{\prime} F_{M(n)} U_{t}^{\prime}$. Now apply 2.5 to $\left(T^{*}, U^{*}, M^{*}\right)$ with $s_{0}=t, A=U_{t}^{\prime}$, to define $U_{s^{*}}^{\prime}$ for all vertices $s^{*}$ of $T^{*}$. This defines $U^{\prime}$ for all vertices of $T$.

Lemma 2.7. Let $(T, U, M)$ be a good tree structure. Let $n \in \mathbb{N}$. Then there is $m>n$ and a refinement $\left(T, U^{\prime}, M\right)$ of $(T, U, M)$ which is good and $F_{n}$ is meager in $F_{m}$ on $\bigcup_{s \in V} U_{s}^{\prime}$, where $V=$ set of vertices of $T$. 
Proof. Since $(T, U, M)$ is good, if $s_{0}$ is a fixed vertex of $T$, we can define by induction on $l\left(s, s_{0}\right)$ a sequence of points $x_{s} \in U_{s}$ with $s \stackrel{n}{-} t \Rightarrow x_{s} F_{M(n)} x_{t}$. By Lemma 2.3 applied to $A=\bigcup_{s \in V} U_{s}$ and the points $\left\{x_{s}\right\}_{s \in V}$, we can find $m>n$ and $A^{*} \subseteq A$ $\Sigma_{1}^{1}$ such that $F_{n}$ is meager in $F_{m}$ on $A^{*}$ and $x_{s} \in A^{*}, \forall s \in V$. Put $U_{s}^{*}=A^{*} \cap U_{s}$. Then $x_{s} \in U_{s}^{*}$ and, by 2.6 applied to $\left(T, U^{*}, M\right)$, we can find a good refinement $\left(T, U^{\prime}, M\right)$ with $x_{s} \in U_{s}^{\prime}$. If $A^{\prime}=\bigcup_{s \in V} U_{s}^{\prime}$, then $F_{n}$ is meager in $F_{m}$ on $A^{\prime}$, since $A^{\prime} \subseteq A^{*}$.

We now come to the final and key lemma. First we need a definition.

Let $T$ be a labelled tree. Given $n \in \mathbb{N}$, we say that two vertices $s, t$ of $T$ are $n$-connected if all the labels in the path from $s$ to $t$ are $\leq n$.

Lemma 2.8. Let $(T, U, M)$ be a good tree structure with $M$ monotone. Let $L$ be the largest label of $T$ and $n \leq L$. Let $N$ be such that $F_{n+1}$ is meager in $F_{N}$ on $\bigcup_{s \in V} U_{s}(V=$ the set of vertices of $T)$, where $M(n) \leq N \leq M\left(n^{\prime}\right)$ for any label $n^{\prime}>n$ (if such exists.) Then there are two refinements $\left(T, U^{0}, M\right),\left(T, U^{1}, M\right)$ of $(T, U, M)$ which are good and

(i) $U_{s}^{0} F_{N} U_{s}^{1}$, for any $s \in V$;

(ii) $\left(U_{s}^{0} \times U_{t}^{1}\right) \cap F_{n+1}=\varnothing$, if $s$, $t$ are $n$-connected.

Proof. Clearly $n$-connectedness is an equivalence relation on $V$, dividing it into components which are subtrees of $T$. Enumerate these as $C_{1}, \ldots, C_{K}$.

We will consider first the case $K=1$, i.e., $n=L$, in which case the requirement $N \leq M\left(n^{\prime}\right), \forall n^{\prime}>n$ is vacuous. So we must have $U_{s}^{0} F_{N} U_{s}^{1}, \forall s \in V$ and $\left(U_{s}^{0} \times U_{t}^{1}\right) \cap F_{n+1}=\varnothing, \forall s, t \in V$.

Enumerate in a sequence $\left(s_{1}, t_{1}\right), \ldots,\left(s_{p}, t_{p}\right)$ the set $V \times V$. We will define by induction on $0 \leq j \leq p$ good tree structures $\left(T, U^{i, j}, M\right)$ for $i=0,1$ such that

A) $U^{i, 0}=U, i \in\{0,1\}$;

and for $j+1 \leq p$ :

B) $\left(T, U^{i, j+1}, M\right)$ refines $\left(T, U^{i, j}, M\right)$;

C) $\left(U^{0, j+1}\left(s_{j+1}\right) \times U^{1, j+1}\left(t_{j+1}\right)\right) \cap F_{n+1}=\varnothing$;

D) $U^{0, j+1}\left(s_{j+1}\right) F_{N} U^{1, j+1}\left(t_{j+1}\right)$.

If this can be done, put $U_{s}^{0}=U^{0, p}(s), U_{s}^{1}=U^{1, p}(s)$. By C), if $(s, t)=$ $\left(s_{j+1}, t_{j+1}\right)$, we have $U_{s}^{0} \times U_{t}^{1}=U^{0, p}(s) \times U^{1, p}(t) \subseteq U^{0, j+1}\left(s_{j+1}\right) \times U^{1, j+1}\left(t_{j+1}\right)$, which is disjoint from $F_{n+1}$. So (ii) is satisfied. For (i), notice that we have $U_{s_{p}}^{0} F_{N} U_{t_{p}}^{1}$. Given any $s \in V$, there is a path from $s$ to $s_{p}$ with labels $\leq L=n$, so by transitivity and the fact that $M$ is monotone and $M(n) \leq N$, we have $U_{s}^{0} F_{N} U_{s_{p}}^{0}$. Similarly, $U_{s}^{1} F_{N} U_{t_{p}}^{1}$, so $U_{s}^{0} F_{N} U_{s}^{1}$.

For the inductive construction of $U^{i, j}$, note that $U^{i, 0}$ is given. Assume $U^{i, j}$ is given for both $i=0,1$ in order to construct $U^{i, j+1}$. Since $F_{n+1}$ is meager in $F_{N}$ on $\left(U^{0, j}\left(s_{j+1}\right) \cup U^{1, j}\left(t_{j+1}\right)\right)$, we can shrink $U^{0, j}\left(s_{j+1}\right), U^{1, j}\left(t_{j+1}\right)$ to nonempty $\Sigma_{1}^{1}$ sets $A, B$ resp., so that $(A \times B) \cap F_{n+1}=\varnothing$ but $A F_{N} B$. (Notice that, by the induction hypothesis, $U^{0, j}\left(s_{j+1}\right) F_{N} U^{1, j}\left(t_{j+1}\right)$.) Then apply 2.5 to $\left(T, U^{0, j}, M\right)$, $A \subseteq U^{0, j}\left(s_{j+1}\right)$ and $\left(T, U^{1, j}, M\right), B \subseteq U^{1, j}\left(t_{j+1}\right)$ to obtain good refinements $\left(T, U^{0, j+1}, M\right),\left(T, U^{1, j+1}, M\right)$ resp., with $U^{0, j+1}\left(s_{j+1}\right)=A, U^{1, j+1}\left(t_{j+1}\right)=B$.

Consider finally the case when $K>1$, i.e., $n<L$. We will define by induction on $1 \leq j \leq K$ good tree structures $\left(T, U^{0, j}, M\right),\left(T, U^{1, j}, M\right)$ such that:

A) $U^{0,0}=U^{1,0}=U$

B) $\left(T, U^{i, j+1}, M\right)$ refines $\left(T, U^{i, j}, M\right)$ for $i=0,1, j<K$;

C) if $s, t \in C_{j^{\prime}}, j^{\prime} \leq j$, then $U^{0, j}(s) F_{N} U^{1, j}(t)$; 
D) if $s, t \in C_{j}$, then $\left(U^{0, j}(s) \times U^{1, j}(t)\right) \cap F_{n+1}=\varnothing$;

E) if $j^{\prime}>j$, then for $s \in C_{j^{\prime}}$, we have $U^{0, j}(s)=U^{1, j}(s)$.

We then put $U_{s}^{0}=U^{0, K}(s), U_{s}^{1}=U^{1, K}(s)$. This clearly works.

We are given $U^{0,0}, U^{1,0}$ by A). Assume now $U^{i, j}$ has been defined for $i=0,1$. We will define $U^{i, j+1}$. Let $C=C_{j+1}$. Then by E) $U^{0, j}\left|C=U^{1, j}\right| C$. Since $F_{n+1}$ is meager in $F_{N}$ on $\bigcup\left\{U^{i, j}(s): s \in C\right\}$, we can apply the previous case (i.e., $K=1$ ) to define $U^{0, j+1}(s), U^{1, j+1}(s)$ for $s \in C$, which are good refinements of $U^{0, j} \mid C$, $U^{1, j} \mid C$ resp., and satisfy C), D) for $s, t \in C$.

We can now use the same argument as in 2.5 to define $U^{0, j+1}(s), U^{1, j+1}(s)$ for $s \notin C$. For such an $s$ there is a unique shortest path to some point in $C$ of length $l(s, C)$. We define $U^{i, j+1}(x)$ inductively on $l(s, C)$ : If $s^{\prime}$ is the next vertex in the shortest path from $s$ to $C$, we can assume by induction that $U^{i, j+1}\left(s^{\prime}\right)$ has been defined and we let

$$
U^{i, j+1}(s)=U^{i, j}(s) \cap\left[U^{i, j+1}\left(s^{\prime}\right)\right]_{F_{M(k)}}
$$

if $s \frac{k}{-} s^{\prime}$.

Clearly B) is satisfied, and so is D), for $j+1$.

To prove $\mathrm{C}$ ), we note that it is clear if $s, t \in C_{j+1}$ by construction. So assume $s, t \in C_{j^{\prime}}, j^{\prime} \leq j$. Since $\left(T, U^{i, j+1}, M\right)$ are good and $N \geq M(n)$, we have, by transitivity, $U^{i, j+1}(s) F_{N} U^{i, j+1}(t)$. So it is enough to show that

$$
U^{0, j+1}(s) F_{N} U^{1, j+1}(s), \forall s \in V .
$$

So we prove, by induction on $l(s, C)$, that $U^{0, j+1}(s) F_{N} U^{1, j+1}(s)$. We let $l(s, C)=0$, if $s \in C$. This is clear then for $l(s, C)=0$, by construction. Else let $s^{\prime}$ be as before, so by the induction hypothesis, $U^{0, j+1}\left(s^{\prime}\right) F_{N} U^{1, j+1}\left(s^{\prime}\right)$. If $k \leq n$ (where $s \frac{k}{s} s^{\prime}$ ), then

$$
U^{0, j+1}(s) F_{M(k)} U^{0, j+1}\left(s^{\prime}\right) F_{N} U^{1, j+1}\left(s^{\prime}\right) F_{M(k)} U^{0, j+1}\left(s^{\prime}\right),
$$

so we are done as $M(k) \leq M(n) \leq N$, by transitivity. Else $k>n$. Then let $x \in U^{0, j+1}(s)$. Since $U^{0, j}(s) F_{N} U^{1, j}(s)$ (by C), E) for $j$ ), let $y \in U^{1, j}(s)$ be such that $x F_{N} y$. Let also $x^{\prime} \in U^{0, j+1}\left(s^{\prime}\right)$ with $x^{\prime} F_{M(k)} x$ and $y^{\prime} \in U^{1, j+1}\left(s^{\prime}\right)$ with $x^{\prime} F_{N} y^{\prime}$. Then $y F_{M(k)} y^{\prime}$, as $N \leq M(k)$, so $y \in U^{1, j+1}(s)$ by definition. So, reversing also the roles of $U^{0, j+1}(s)$ and $U^{1, j+1}(s)$, we have $U^{0, j+1}(s) F_{N} U^{1, j+1}(s)$.

Finally, we prove E), i.e.,

$$
s \in C_{j^{\prime}}, j^{\prime}>j+1 \Rightarrow U^{0, j+1}(s)=U^{1, j+1}(s) .
$$

This is again by induction on $l(s, C)$. Let $s^{\prime}$ be as before, $s \frac{k}{s} s^{\prime}$, and assume $(*)$ holds for $s^{\prime}$. If $s^{\prime} \in C_{j^{\prime}}, j^{\prime}>j+1$, then we are clearly done, since

$$
\begin{aligned}
U^{0, j+1}(s) & =U^{0, j}(s) \cap\left[U^{0, j+1}\left(s^{\prime}\right)\right]_{F_{M(k)}} \\
& =U^{1, j}(s) \cap\left[U^{1, j+1}\left(s^{\prime}\right)\right]_{F_{M(k)}}
\end{aligned}
$$

(by the induction hypothesis for $s^{\prime}$ and E) for $j$ )

$$
=U^{1, j+1}(s) .
$$

Otherwise, $s \in C_{j^{\prime}}$, for $j^{\prime} \leq j+1$, so that in particular $k>n$, and thus $M(k) \geq N$. Then, by C),

$$
U^{0, j+1}\left(s^{\prime}\right) F_{N} U^{1, j+1}\left(s^{\prime}\right)
$$


SO

$$
U^{0, j+1}\left(s^{\prime}\right) F_{M(k)} U^{1, j+1}\left(s^{\prime}\right),
$$

i.e.,

$$
\left[U^{0, j+1}\left(s^{\prime}\right)\right]_{F_{M(k)}}=\left[U^{1, j+1}\left(s^{\prime}\right)\right]_{F_{M(k)}},
$$

and we are done as above.

We are now ready to proceed to the construction of $U_{s}$ for $s \in 2^{p+1}$ satisfying $R(1)-(4)$, assuming $U_{s}$, for $s \in \bigcup_{p^{\prime} \leq p} 2^{p^{\prime}}$, are given satisfying $R(1)-(4)$ for all $p^{\prime}<p$.

Lemma 2.9. Let $A$ be a finite set, $\sim_{0} \subseteq \sim_{1} \subseteq \cdots \subseteq \sim_{k}$ a sequence of equivalence relations on $A$, with $\sim_{0}=$ equality and $\sim_{k}=A \times A$. Then there is a labelled tree $T$ with set of vertices $A$ and labels in the set $\{0, \ldots, k\}$ such that

$$
a \sim_{j} b \Leftrightarrow a, b \text { are } j \text {-connected. }
$$

Proof. By induction on $k$. For $k=0$, this is obvious. Assume it true for $k=p$. Let $k=p+1$. Pick a point $a_{i} \in C_{i}$, where $\left\{C_{i}\right\}_{i=1}^{q}$ are the $\sim_{p^{-}}$equivalence classes. For each $C_{i}$ there is, by the induction hypothesis, a labelled tree $T_{i}$ with set of vertices $C_{i}$ and labels $\{0, \ldots, p\}$ satisfying the above for $\sim_{j} \mid C_{i}, 0 \leq j \leq p$. Define $T$, with set of vertices $A$, by adding to the edges of the $T_{i}$ 's the edges $\left(a_{i}, a_{i+1}\right)$ for $i=1, \ldots, q-1$ with label $p+1=k$. This clearly works.

Apply this lemma now to $A=2^{p}$ and $\sim_{0}, \sim_{1}, \ldots, \sim_{L(p)}$. Call the resulting labelled tree $T$. Consider the tree structure $(T, U, M)$, where $U$ is as given by the induction hypothesis and $M(n)=N(n)$ for $n \leq L(p)$, which again is given by the induction hypothesis. Note that $M$ is monotone (in fact strictly increasing).

Note now that condition $R(4)$ for $p^{\prime}=p-1$ implies that $(T, U, M)$ is good: Indeed, let $p^{\prime}=\left\langle n^{\prime}, k^{\prime}\right\rangle$. Let $\bar{s}, \bar{t} \in 2^{p}$ be such that $\bar{s}=\widehat{s} i, \bar{t}=t^{\prime} i^{\prime}, s, t \in 2^{p^{\prime}}$. Let $j \leq L(p)=L\left(p^{\prime}+1\right)$ be such that $\bar{s} \sim_{j} \bar{t}$. There are two cases: (A) $k^{\prime}=0$, so that $L\left(p^{\prime}\right)=n^{\prime}, L\left(p^{\prime}+1\right)=L(p)=n^{\prime}+1$; (B) $k^{\prime}>0$, so that $n^{\prime}<L\left(p^{\prime}\right)=L\left(p^{\prime}+1\right)$. In case (A), if $j=L(p)=n^{\prime}+1$ then $U_{\bar{s}} F_{N(j)} U_{\bar{t}}$ by $R(4)$ (a), (b) for $p^{\prime}$, transitivity, and the monotonicity of $N$. If $j<L(p)$, i.e., $j \leq n^{\prime}$, then $i=i^{\prime}$, so since also $j \leq L\left(p^{\prime}\right)$, we have by $R(4)$ (a) for $p^{\prime}$ that $U_{\bar{s}} F_{N(j)} U_{\bar{t}}$. In case (B), if $j=L(p)$ we are done as before. If $j<L(p)=L\left(p^{\prime}\right)$, then either $i=i^{\prime}$, and since $s \sim_{j} t$ as well, we are done, by $R(4)$ (a) for $p^{\prime}$; or else $i \neq i^{\prime}$ in which case $j>n^{\prime}$, so $N(j) \geq N\left(n^{\prime}+1\right)$. Again $s \sim_{j} t$, so $U_{s^{\wedge} i} F_{N(j)} U_{t^{\imath} i}$ by $R(4)$ (a) for $p^{\prime}$ and $U_{t^{\wedge} i^{\prime}} F_{N\left(n^{\prime}+1\right)} U_{t^{\wedge} i}$ by $R(4)$ (b) for $p^{\prime}$, thus $U_{s^{\wedge} i} F_{N(j)} U_{t^{\wedge} i^{\prime}}$ again.

We now have two cases for $p$.

Case $(\alpha): p=\langle n, 0\rangle$, so that $L(p)=n$ and $L(p+1)=n+1$.

In this case we have to define also $N(n+1)$. For that we apply 2.7: We can find a refinement $\left(T, U^{*}, M\right)$ of $(T, U, M)$ and $N(n+1)>N(n)$ such that $F_{n+1}$ is meager in $F_{N(n+1)}$ on $\bigcup_{s \in 2^{p}} U_{s}^{*}$. (Note that if $F_{n+1}$ is meager in $F_{m}$ on $A$, it is also meager in $F_{m^{\prime}}$ on $A$ for $m^{\prime} \geq m$.) By applying also 2.5 , repeatedly, we can assume that $d\left(U_{s}^{*}\right) \leq 2^{-p}$ and $\overline{U_{s}^{*}} \subseteq U_{s}$.

Case $(\beta): p=\langle n, k\rangle$ for $k>0$, so that $n<L(p)=L(p+1)$.

In this case, we do not have to define a new value of $N$. Also, by the induction hypothesis, $R(3)$ implies that $F_{n+1}$ is meager $\operatorname{in} F_{N(n+1)}$ on $\bigcup_{s \in 2^{p}} U_{s}$. So let $\left(T, U^{*}, M\right)$ be a good refinement of $(T, U, M)$ so that $d\left(U_{s}^{*}\right) \leq 2^{-p}$ and ${\overline{U_{s}^{*}}}^{\tau} \subseteq U_{s}$ (by 2.5 again). 
So in either case we have a good tree structure $\left(T, U^{*}, M\right)$ refining $(T, U, M)$ with $F_{n+1}$ meager in $F_{N(n+1)}$ on $\bigcup_{s \in 2^{p}} U_{s}^{*}$ and $d\left(U_{s}^{*}\right) \leq 2^{-p},{\overline{U_{x}^{*}}}^{\tau} \subseteq U_{s}$. Let $N=$ $N(n+1)$. Thus $M(n) \leq N \leq M\left(n^{\prime}\right)$ for any $n^{\prime}>n, n^{\prime} \leq L(p)$ (if such exists). By 2.8 then, there are good refinements $\left(T, U^{0}, M\right),\left(T, U^{1}, M\right)$ of $\left(T, U^{*}, M\right)$ satisfying $U_{s}^{0} F_{N} U_{s}^{1}, \forall s \in 2^{p}$ and $\left(U_{s}^{0} \times U_{t}^{1}\right) \cap F_{n+1}=\varnothing$, for any two vertices $s, t$ of $T$ which are $n$-connected, i.e., by $2.9, s \sim_{n} t$. We put now

$$
U_{s^{\wedge} i}=U_{s}^{i} .
$$

Clearly $R(1)$, (2) are satisfied (note that $U_{s^{\wedge} 0} \cap U_{s^{\wedge} 1}=\varnothing$ follows from $R(2)$ ). Moreover, $R(3)$ holds by the choice of $N(n+1)$ and the induction hypothesis. Next, $R(4)$ (a) is true since $\left(T, U_{s}^{0}, M\right),\left(T, U_{s}^{1}, M\right)$ are good and $M$ is monotone. Finally, $R(4)$ (b) holds because of (i) of 2.8 .

Thus the construction is complete, and so is the proof of 2.2

Let us note also the following corollary of the main result, which points out another interesting property of $E_{1}$.

Theorem 2.10. Let $E$ be a Borel equivalence relation. Then

$$
E_{1} \leq E \Leftrightarrow E_{1} \sqsubseteq^{i} E .
$$

Proof. Let $f:\left(2^{\mathbb{N}}\right)^{\mathbb{N}} \rightarrow X$ be such that $x E_{1} y \Leftrightarrow f(x) E f(y)$. Let $X^{*}=f\left[\left(2^{\mathbb{N}}\right)^{\mathbb{N}}\right]$ and $X^{* *}=\left[X^{*}\right]_{E}$. We claim first that $X^{* *}$ is Borel. Indeed, let

$$
R(x, y) \Leftrightarrow f(y) E x .
$$

Then $R$ is Borel with $K_{\sigma}$ sections, so since $x \in X^{* *} \Leftrightarrow \exists y R(x, y), X^{* *}$ is Borel. Moreover, there is a Borel function $\varphi: X^{* *} \rightarrow\left(2^{\mathbb{N}}\right)^{\mathbb{N}}$ such that $R(x, \varphi(x))$, i.e., $f \circ$ $\varphi(x) E x$. Then $\varphi$ is a reduction of $E \mid X^{* *}$ into $E_{1}$, so from 2.1 it follows that $E_{1} \sqsubseteq E \mid X^{* *}$; thus $E_{1} \sqsubseteq E$. So we can assume above that $f$ is $1-1$. We can also suppose that $X=2^{\mathbb{N}}$.

Now define $g: X^{* *} \rightarrow\left(2^{\mathbb{N}}\right)^{\mathbb{N}}$ by $g(x)=\left(x, \varphi(x)_{1}, \varphi(x)_{2}, \ldots\right)$. Then $g$ is $1-1$ and $g(x) E_{1} \varphi(x)$, so $f \circ g(x) E x$. Also $g \circ f(y) E_{1} y$. Now apply Schroeder-Bernstein to $f, g$ to show that $E_{1} \cong E_{1} \mid X^{* *}$, thus $E_{1} \sqsubseteq^{i} E$.

\section{Stable EQUivalenCE AND ISOMORPHISM}

As an application of the result in Section 2 we can also classify hypersmooth Borel equivalence relations, at least under further mild regularity assumptions, with respect to stable equivalence, where we call two Borel equivalence relations $E, F$ stably equivalent if

$$
E \times I_{2^{\mathbb{N}}} \cong F \times I_{2^{\mathbb{N}}}
$$

Let us say that a Borel equivalence relation $E$ on $X$ is strongly smooth if it admits a Borel selector, and strongly hypersmooth if $E=\bigcup_{n} F_{n}$, with $F_{0} \subseteq$ $F_{1} \subseteq \cdots$ and $F_{n}$ strongly smooth.

There are easy examples of smooth Borel $E$ which are not strongly smooth (see, e.g., [K3, 18.D]), and from 3.8 below these are not even strongly hypersmooth. However most natural examples of smooth $E$ are actually strongly smooth. Also every smooth $E$ with $K_{\sigma}$ equivalence classes is strongly smooth. 
We have

Theorem 3.1. Let $E$ be a non-smooth strongly hypersmooth Borel equivalence relation. Then $E$ is stably equivalent to exactly one of $E_{0} \times I_{2^{\mathbb{N}}}, E_{1}$.

The same conclusion holds if $E$ is hypersmooth with $K_{\sigma}$ equivalence classes, in the sense that $E$ is a Borel equivalence relation on a Borel set $B$ in some Polish space $Y$ and all E-equivalence classes are $K_{\sigma}$ in $Y$.

Proof. We will need the following two lemmas:

Lemma 3.2. Let $E$ be a Borel equivalence relation with $K_{\sigma}$ classes, i.e., $E$ is a Borel equivalence relation on a Borel set $B$ in a Polish space $Y$ and each $E$ equivalence class is $K_{\sigma}$ in $Y$. Let $X$ be a standard Borel space and $F$ a Borel equivalence relation on $X$. If $E \times I_{2^{\mathbb{N}}} \sqsubseteq F$, then $E \times I_{2^{\mathbb{N}}} \sqsubseteq^{i} F$.

Proof. Let the Borel function $f$ embed $E \times I_{2^{\mathbb{N}}}$ into $F$ and define $X^{*}, X^{* *}$ as in the proof of 2.10. As in that proof, $X^{* *}$ is Borel and there is a Borel function $\varphi$ : $X^{* *} \rightarrow B \times 2^{\mathbb{N}}$ with $f \circ \varphi(x) F x$. Assuming, without loss of generality, that $X=2^{\mathbb{N}}$, define $g: X^{* *} \rightarrow B \times 2^{\mathbb{N}}$ as follows: If $\varphi(x)=(b, z)$, then $g(x)=(b, x)$. Then $\varphi(x) E \times I_{2^{\mathbb{N}}} g(x)$, so $f \circ g(x) F x$. Also $g$ is 1-1. Now apply Schroeder-Bernstein.

Lemma 3.3. Let $E$ be Borel and strongly hypersmooth. Then $E \times I_{2^{\mathbb{N}}} \sqsubseteq^{i} E_{1}$.

Proof. Put $\widetilde{E}=E \times I_{2^{\mathbb{N}}}$, so that $\widetilde{E}$ is also strongly hypersmooth. We can of course assume that the space of $\widetilde{E}$ is $2^{\mathbb{N}}$. Let $\widetilde{E}=\bigcup_{n} F_{n}, F_{0} \subseteq F_{1} \subseteq \cdots$, with $F_{0}=\Delta\left(2^{\mathbb{N}}\right), F_{n}$ strongly smooth with Borel selector $f_{n}$. Consider the canonical embedding $f(x)=\left(f_{n}(x)\right)$ of $\widetilde{E}$ into $E_{1}$, and define $X^{*}, X^{* *}$ for this $f$ as in the proof of 2.10. We claim that $X^{* *}$ is Borel and there is Borel $\varphi: X^{* *} \rightarrow 2^{\mathbb{N}}$ with $f \circ \varphi\left(\left(x_{m}\right)\right) E_{1}\left(x_{m}\right)$. The proof then can be completed as in 3.2.

To see that $X^{* *}$ is Borel, we verify that

$$
\left(x_{m}\right) \in X^{* *} \Leftrightarrow \exists n \forall m \geq n\left(x_{m}=f_{m}\left(x_{n}\right)\right) .
$$

$\Rightarrow$ : Pick $x$ so that $\left(x_{m}\right) E_{1} f(x)$ and $n$ so that $\forall m \geq n\left(x_{m}=f_{m}(x)\right)$. Then $x_{n}=f_{n}(x)$ and $x_{n} F_{n} x$, so $x_{n} F_{m} x$ for $m \geq n$ and $f_{m}\left(x_{n}\right)=f_{m}(x)$ for $m \geq n$; thus $x_{m}=f_{m}\left(x_{n}\right), \forall m \geq n$.

$\Leftarrow$ : Fix $n$ with $x_{m}=f_{m}\left(x_{n}\right)$ for $m \geq n$. Then $f\left(x_{n}\right) E_{1}\left(x_{m}\right)$ and $\left(x_{m}\right) \in X^{* *}$.

Finally, if $\left(x_{m}\right) \in X^{* *}$, let

$$
\varphi\left(\left(x_{m}\right)\right)=x_{n},
$$

where $n$ is least with $\forall m \geq n\left(x_{m}=f_{m}\left(x_{n}\right)\right)$. Then $f \circ \varphi((m)) E_{1}\left(x_{m}\right)$.

We now complete the proof of 3.1. Since $E$ is not smooth, $E_{0} \sqsubseteq E$, so $E_{0} \times I_{2^{\mathbb{N}}} \sqsubseteq$ $E \times I_{2^{\mathbb{N}}}$. Since $E_{0} \times I_{2^{\mathbb{N}}}$ has $K_{\sigma}$ equivalence classes and $E_{0} \times I_{2^{\mathbb{N}}} \times I_{2^{\mathbb{N}}} \cong E_{0} \times I_{2^{\mathbb{N}}}$, we have by 3.2 that $E_{0} \times I_{2^{\mathbb{N}}} \sqsubseteq^{i} E \times I_{2^{\mathbb{N}}}$.

Since $E$ is hypersmooth, $E \leq E_{0}$ or else $E_{1} \sqsubseteq E$. In the first case we have that $E \times I_{2^{\mathbb{N}}} \sqsubseteq E_{0} \times I_{2^{\mathbb{N}}}$. (Indeed, if $g$ reduces $E$ to $E_{0}$, the function

$$
f(x, \alpha)=(g(x),\langle x, \alpha\rangle),
$$

where \langle\rangle$: X \times 2^{\mathbb{N}} \rightarrow 2^{\mathbb{N}}$ is a Borel injection and $X$ the space of $E$, embeds $E \times I_{2^{\mathbb{N}}}$ into $E_{0} \times I_{2^{\mathbb{N}}}$.) If now $E$ has $K_{\sigma}$ equivalence classes, $E \times I_{2^{\mathbb{N}}} \sqsubseteq^{i} E_{0} \times I_{2^{\mathbb{N}}}$ by 3.2 , so $E \times I_{2^{\mathbb{N}}} \cong E_{0} \times I_{2^{\mathbb{N}}}$. On the other hand, if $E$ is strongly hypersmooth, $E \times I_{2^{\mathbb{N}}} \sqsubseteq^{i} E_{1}$ by 3.3, so, replacing $E \times I_{2^{\mathbb{N}}}$ by an isomorphic copy, we can assume that it has $K_{\sigma}$ equivalence classes, so, by 3.2 again, $E \times I_{2^{\mathbb{N}}} \sqsubseteq^{i} E_{0} \times I_{2^{\mathbb{N}}}$ and we have shown that $E \times I_{2^{\mathbb{N}}} \cong E_{0} \times I_{2^{\mathbb{N}}}$. 
In the case when $E_{1} \sqsubseteq E$, we have by 2.10 that $E_{1} \sqsubseteq^{i} E \times I_{2^{\mathrm{N}}}$. By 1.3 and 3.2 or 3.3, depending on whether $E$ has $K_{\sigma}$ equivalence classes or is strongly hypersmooth, we also have $E \times I_{2^{\mathbb{N}}} \sqsubseteq^{i} E_{1}$, so $E_{1} \cong E \times I_{\mathbb{R}}$.

Let us call a Borel equivalence relation $E$ uniformly continuous if $E \cong E \times I_{2^{\mathrm{N}}}$. In view of 3.1, the only uniformly continuous, non-smooth, strongly hypersmooth Borel equivalence relations are $E_{0} \times I_{2^{\mathrm{N}}}$ and $E_{1}$. This should be compared with analogous measure theoretic results of Vershik and Vinokurov-Ganikhodzhaev (see $[\mathrm{V}],[\mathrm{VF}],[\mathrm{VG}]$.

The following criterion can be useful in verifying whether a given $E$ is uniformly continuous.

Proposition 3.4. Let $E$ be a Borel equivalence relation. Then the following are equivalent:

(i) $E$ is uniformly continuous.

(ii) There is a smooth Borel $F \subseteq E$ which has uniformly continuum-size equivalence classes, i.e., there is a Borel function $f: X \times 2^{\mathbb{N}} \rightarrow X$ such that $x F y \rightarrow$ $f(x, \alpha)=f(y, \alpha) F x$ and $\alpha \neq \beta \Rightarrow f(x, \alpha) \neq f(x, \beta)$ (where $X$ is the space of $E$ ).

Proof. (i) $\Rightarrow$ (ii): It is enough to show that $E \times I_{2^{\mathrm{N}}}$ satisfies (ii). Indeed, let $F \subseteq$ $E \times I_{2^{\mathbb{N}}}$ be given by

$$
(x, \alpha) F(y, \beta) \Leftrightarrow x=y .
$$

Clearly $F$ is smooth. Put also

$$
f((x, \alpha), r)=(x, r) .
$$

(ii) $\Rightarrow\left(\right.$ i): Let $g(x)=(x, \overline{0})$, so that $g$ embeds $E$ into $E \times I_{2^{\mathbb{N}}}($ where $\overline{0}=000 \cdots)$. Let $f$ be given now by (ii). Fix a Borel injection \langle\rangle$: X \times 2^{\mathbb{N}} \rightarrow 2^{\mathbb{N}}$ and define $h: X \times 2^{\mathbb{N}} \rightarrow 2^{\mathbb{N}}$ by

$$
h(x, \alpha)=f(x,\langle x, \alpha\rangle) .
$$

Then $h$ is $1-1$ and, since $h(x, \alpha) E x$,

$$
g \circ h(x, \alpha) E \times I_{2^{\mathbb{N}}}(x, \alpha),
$$

while

$$
h \circ g(x) E x,
$$

so by applying Schroeder-Bernstein to $g, h$ we have that $E \cong E \times I_{2^{\mathrm{N}}}$.

As an application, we see that

$$
E_{1}\left(=E_{0}\left(2^{\mathbb{N}}\right)\right) \cong E_{t}\left(2^{\mathbb{N}}\right) .
$$

This is because $E_{t}\left(2^{\mathbb{N}}\right)$ is hypersmooth, uniformly continuous (as it contains $F$, where $x F y \Leftrightarrow \forall n \geq 1\left(x_{n}=y_{n}\right)$ ) and $E_{1} \sqsubseteq E_{t}\left(2^{\mathbb{N}}\right)$ (via the map $x \in\left(2^{\mathbb{N}}\right)^{\mathbb{N}} \mapsto$ $\left(\left\langle\bar{n}, x_{n}\right\rangle\right) \in\left(2^{\mathbb{N}}\right)^{\mathbb{N}}$, where $\bar{n}=0^{n \wedge} 1^{\infty},\langle\rangle:\left(2^{\mathbb{N}}\right)^{2} \rightarrow 2^{\mathbb{N}}$ is a Borel injection); thus $E_{t}\left(2^{\mathbb{N}}\right) \not \leq E_{0}$. In fact, more generally, if $E$ is Borel hypersmooth with $E_{1} \subseteq E \subseteq E_{C}^{\prime}$, where $E_{C}^{\prime}$ on $\left(2^{\mathbb{N}}\right)^{\mathbb{N}}$ is defined by

$$
\left(x_{n}\right) E_{C}^{\prime}\left(y_{n}\right) \Leftrightarrow\left\{x_{n}: n \in \mathbb{N}\right\} \Delta\left\{y_{n}: n \in \mathbb{N}\right\} \text { is finite, }
$$

then $E \cong E_{1}$. This is because any such $E$ is not reducible to a countable Borel equivalence relation, by 1.5 , and is uniformly continuous, since it contains $F$ as above. 


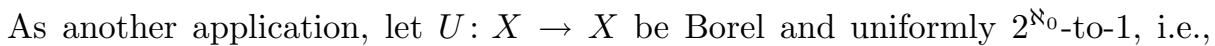
assume there is a function $V: U[X] \times 2^{\mathbb{N}} \rightarrow X$ with analytic graph such that $U(V(y, \alpha))=y$ for all $\alpha \in 2^{\mathbb{N}}$, and $\alpha \neq \beta \Rightarrow V(y, \alpha) \neq V(y, \beta)$. Then $E_{0}(U), E_{t}(V)$ are either smooth or else Borel isomorphic to one of $E_{1}, E_{0} \times I_{2^{\mathrm{N}}}$. This is because $F \subseteq E_{0}(U) \subseteq E_{t}(U)$, where $x F y \Leftrightarrow U(x)=U(y)$, and $F$ together with $f(x, \alpha)=$ $V(U(x), \alpha)$ satisfies 3.4, (ii). (Of course, all these cases can occur, as we can see by taking $U$ to be the restriction of the shift on $\left(2^{\mathbb{N}}\right)^{\mathbb{N}}$ to various Borel invariant subsets.)

In [K2] it is shown that for any Borel equivalence relation of the form $E_{\mathbb{R}}$ (i.e., induced by a Borel flow) none of whose equivalence classes is a singleton, we have that either $E_{\mathbb{R}}$ is smooth or else $E_{\mathbb{R}} \cong E_{0} \times I_{2^{\mathbb{N}}}$. By the results in [JKL] it follows also that if $G$ is compactly generated of polynomial growth and $E_{G}$ has all equivalence classes uncountable, then again $E_{G}$ is smooth or else $E_{G} \cong E_{0} \times I_{2^{\mathrm{N}}}$.

Finally, we can apply also the preceding methods to classify $E_{H}$, and therefore the coset spaces $G / H$, for subgroups $H$ of Polish groups $G$, which can be written as unions of increasing sequences of closed subgroups. The result is as follows:

Theorem 3.5. Let $G$ be a Polish group, $H=\bigcup_{n} H_{n}$, where $\left(H_{n}\right)$ is a increasing sequence of closed subgroups. Denote by $E_{H}$ the equivalence relation

$$
x E_{H} y \Leftrightarrow x H=y H .
$$

Then exactly one of the following holds:

(i) $H$ is closed and $E_{H}$ is smooth.

(ii) $H$ is not closed but, for sufficiently large $n, H_{n+1} / H_{n}$ is countable. Then if $H$ is uncountable, $E_{H} \cong E_{0} \times I_{2^{\mathbb{N}}}$, while if $H$ is countable $E_{H} \approx E_{0}$.

(iii) For infinitely many $n, H_{n+1} / H_{n}$ is uncountable and $E_{H} \cong E_{1}$.

Proof. Assume that $H$ is not closed, so $E_{H}$ is not smooth. Then $E_{0} \sqsubseteq E_{H}$.

Consider first the case where for sufficiently large $n, H_{n+1} / H_{n}$ is countable. If all $H_{n}$ are countable, so is $H$ and thus $E_{H}$ is countable. Since $E_{H}$ is hypersmooth, by [DJK], $E_{H}$ is then hyperfinite and $E_{H} \approx E_{0}$. So assume some $H_{n}$ is uncountable. Renumber so that $H_{0}$ is uncountable and $H_{n+1} / H_{n}$ is countable for all $n$, i.e., $H_{n} / H_{0}$ is countable for all $n$ and so $H / H_{0}$ is countable. Then $E_{H_{0}}$ is strongly smooth and $E_{H} / E_{H_{0}}$ is countable. It follows that $E_{H} \leq E_{0}$. Let $X_{0}$ be a Borel transversal for $E_{H_{0}}$. Define $F_{n}$ on $X_{0}$ by

$$
F_{n}=E_{H_{n}}\left\lceil X_{0}\right.
$$

and $F=\bigcup_{n} F_{n}$, so $F=E_{H} \uparrow X_{0}$. Since $F$ is countable Borel and hypersmooth, it is hyperfinite, so $F \leq E_{0}$. But $E_{H} \leq F$ by the map: $h(x)=$ unique element of $X_{0} \cap[x]_{E_{H_{0}}}$.

By 3.1 then, $E_{H} \times I_{\mathbb{R}} \cong E_{0} \times I_{\mathbb{R}}$. But we claim that $E_{H}$ is uniformly continuous, so $E_{H} \cong E_{0} \times I_{\mathbb{R}}$. For that we use 3.4. Let $F=E_{H_{0}}$. Since $H_{0}$ is uncountable, let $\varphi: \mathbb{R} \rightarrow H_{0}$ be a Borel bijection. Let $X_{0}$ be a Borel transversal for $E_{H_{0}}$ and let $h(x)$ be defined as above. Put $f(x, \alpha)=h(x) \cdot \varphi(\alpha)$.

Now consider the case when for infinitely many $n, H_{n+1} / H_{n}$ is uncountable. By renumbering, we can assume that $H_{0}$ is uncountable and $H_{n+1} / H_{n}$ is uncountable for each $n$. We will show then that $E_{1} \sqsubseteq E_{H}$. As before $E_{H}$ is strongly hypersmooth and uniformly continuous, so $E_{H} \cong E_{1}$.

Since $H_{n+1} / H_{n}$ is uncountable, we claim that $H_{n}$ is meager in $H_{n+1}$. Indeed, otherwise $H_{n}=H_{n} \cdot H_{n}^{-1}$ would contain an open nbhd of the identity in $H_{n+1}$, so $H_{n}$ would be open in $H_{n+1}$ and $H_{n+1} / H_{n}$ would be countable. 
From this we can derive the following.

Lemma. For each $n, E_{H_{n}}$ is meager in $E_{H_{n+1}}$ (equipped with the relativized product topology from $G^{2} ; E_{H_{n+1}}$ is closed in $G^{2}$ ).

Proof. Put $F_{n}=E_{H_{n+1}}$. Let $U, V$ be open in $G$ with $(U \times V) \cap F_{n+1} \neq \varnothing$. We will find open $U^{\prime} \subseteq U, V^{\prime} \subseteq V$ such that $\left(U^{\prime} \times V^{\prime}\right) \cap F_{n+1} \neq \varnothing$, but $\left(U^{\prime} \times V^{\prime}\right) \cap F_{n}=\varnothing$.

Consider $L=\{g \in G: \exists x \in U(x g \in V)\}$. This is open in $G$ and $L \cap H_{n+1} \neq \varnothing$. So find open $L^{\prime} \subseteq L$ with $L^{\prime} \cap H_{n+1} \neq \varnothing$, but $L^{\prime} \cap H_{n}=\varnothing$. Fix $g_{0} \in H_{n+1}, g_{0} \in L^{\prime}$ and $x_{0} \in U$ with $x_{0} g_{0}=y_{0} \in V$. Let

$$
T=\left\{(x, y): x \in U \& y \in V \& x^{-1} y \in L^{\prime}\right\} .
$$

This is an open nbhd of $\left(x_{0}, y_{0}\right)$, so we can find $U^{\prime} \subseteq U, V^{\prime} \subseteq V$ with $\left(x_{0}, y_{0}\right) \in$ $U^{\prime} \times V^{\prime}, U^{\prime} \times V^{\prime} \subseteq T$. Since $\left(x_{0}, y_{0}\right) \in F_{n+1}$, we have $\left(U^{\prime} \times V^{\prime}\right) \cap F_{n+1} \neq \varnothing$. If $\left(U^{\prime} \times V^{\prime}\right) \cap F_{n} \neq \varnothing$, let $x^{\prime} \in U^{\prime}, y^{\prime} \in V^{\prime}, g^{\prime} \in H_{n}$ be such that $x^{\prime} g^{\prime}=y^{\prime}$. Then $\left(x^{\prime}\right)^{-1} y^{\prime}=g^{\prime} \in L^{\prime} \cap H_{n}$, a contradiction. So $\left(U^{\prime} \times V^{\prime}\right) \cap F_{n}=\varnothing$.

We can now repeat the argument of Case II in the proof of 2.1. Instead of the Gandy-Harrington topology we work with the Polish topology of $G$. The two relevant points are:

(i) $E_{H_{n}}$ is meager in $E_{H_{m}}$ for any $n<m$.

(ii) The $E_{H_{n}}$-saturation of an open set in $G$ is also open, being a union of translates of this open set.

Thus in the construction of CaseII we can take $U_{s}$ to be open sets in $G$.

If we are wiling to allow a wider class of isomorphisms than Borel, we actually have a simpler formulation of the preceding results. Recall that a function is $\mathbf{C}$ measurable if it is measurable with respect to the smallest $\sigma$-algebra containing the Borel sets and closed under the Souslin operation $\mathcal{A}$. We also call sets in this class C-measurable.

Theorem 3.6. Let $E$ be a nonsmooth, hypersmooth Borel equivalence relation. If every E-equivalence class is uncountable, then $E$ is isomorphic by a $C$-measurable isomorphism to exactly one of $E_{1}$ or $E_{0} \times I_{2^{\mathrm{N}}}$.

(Here, to say that an isomorphism $f$ between standard Borel spaces is $C$-measurable, means that both $f$ and $f^{-1}$ are $C$-measurable.)

Proof. By 3.1 it is enough to show that if $E$ is as in the hypothesis of 3.6, then $E$ is isomorphic to $E \times I_{2^{\mathrm{N}}}$ by a $C$-measurable isomorphism.

Let $X, Y$ be standard Borel spaces. A bijection $f: A \rightarrow B$, where $A \subseteq X, B \subseteq Y$ and $f[A]=B$, will be called $C$-measurable if $f, f^{-1}, A, B$ are $C$-measurable. Note that by the usual Schroeder-Bernstein argument if $f: X \rightarrow A \subseteq Y, g: Y \rightarrow B \subseteq X$ are $C$-measurable bijections, then there is a $C$-measurable bijection $h: X \rightarrow Y$.

Consider now $E($ on $X)$ and $E \times I_{2^{\mathbb{N}}}\left(\right.$ on $\left.X \times 2^{\mathbb{N}}\right)$. Clearly the map $f(x)=(x, \overline{0})$ is a $C$-measurable bijection of $X$ with $X \times\{\overline{0}\}$. If we can find a $C$-measurable bijection $g: X \times 2^{\mathbb{N}} \longmapsto A \subseteq X$ such that $g(x, \alpha) E x$, then by applying Schroeder-Bernstein to $f, g$ we obtain a $C$-measurable isomorphism of $E$ with $E \times I_{2^{\mathrm{N}}}$.

Let $E=\bigcup_{n} F_{n}, F_{0} \subseteq F_{1} \subseteq \cdots$, where $F_{0}$ is equality and $F_{n}$ is smooth. Let $g_{n}: X \rightarrow X$ be a $C$-measurable selector for $F_{n}$. Define a new $C$-measurable selector $f_{n}$ of $F_{n}$ by letting $f_{0}=$ identity, $f_{n+1}=f_{n} \circ g_{n+1}$. If $T_{n}=\left\{x: f_{n}(x)=x\right\}$ is the corresponding transversal for $f_{n}$, then $T_{n}$ is $C$-measurable and $T_{n+1} \subseteq T_{n}$. 
Lemma 3.7. Let $A_{n}=\left\{x: x \in T_{n} \&[x]_{F_{n}}\right.$ is uncountable $\}$. Then there is a $C$-measurable bijection $R_{n}: A_{n} \times 2^{\mathbb{N}} \rightarrow B_{n} \subseteq X$ such that

(i) $R_{n}(x, \alpha) F_{n} x$,

(ii) $B_{n} \cap B_{m}=\varnothing$, if $n \neq m$.

Granting this lemma, we complete the proof as follows: For each $x \in X$, let $n(x)=$ least $n$ such that $[x]_{F_{n}}$ is uncountable. This exists as $[x]_{E}=\bigcup_{n}[x]_{F_{n}}$ and $[x]_{E}$ is uncountable. Put

$$
g(x, \alpha)=R_{n(x)}\left(f_{n(x)}(x),\langle x, \alpha\rangle\right)
$$

(where \langle\rangle$: X \times 2^{\mathbb{N}} \rightarrow 2^{\mathbb{N}}$ is a Borel bijection.)

First we check that $g$ is 1-1: If $g(x, \alpha)=g\left(x^{\prime}, \alpha^{\prime}\right)$, then as the ranges of $R_{n}$ are disjoint, $n(x)=n\left(x^{\prime}\right)\left(=n\right.$.) So $R_{n}\left(f_{n}(x),\langle x, \alpha\rangle\right)=R_{n}\left(f_{n}\left(x^{\prime}\right),\left\langle x^{\prime}, \alpha^{\prime}\right\rangle\right)=y$. Then $\langle x, \alpha\rangle=\left\langle x^{\prime}, \alpha^{\prime}\right\rangle$.

Clearly $G$ is $C$-measurable -note that $x \mapsto n(x)$ is $C$-measurable. Let $B=$ range $(g)$. We check that $B$ is $C$-measurable: For $\alpha \in 2^{\mathbb{N}}$, let $\alpha=\left\langle(\alpha)_{0},(\alpha)_{1}\right\rangle$. Then if $\pi_{0}, \pi_{1}$ are the two projections of $X \times 2^{\mathbb{N}}$,

$$
\begin{aligned}
y \in \operatorname{range}(g) \Leftrightarrow \exists n[y & \in \operatorname{range}\left(R_{n}\right) \& \\
& n\left(\left(\pi_{1}\left(R_{n}^{-1}(y)\right)\right)_{0}\right)=n \& \\
& \left.\pi_{0}\left(R_{n}^{-1}(y)\right)=f_{n}\left(\left(\pi_{1}\left(R_{n}^{-1}(y)\right)\right)_{0}\right)\right] .
\end{aligned}
$$

Also for $y \in \operatorname{range}(g), A \subseteq X \times \mathbb{R}, A$ a Borel set,

$$
\begin{aligned}
g^{-1}(y) \in A \Leftrightarrow \exists n[y & \in \operatorname{range}\left(R_{n}\right) \& \\
& n\left(\left(\pi_{1}\left(R_{n}^{-1}(y)\right)\right)_{0}\right)=n \& \\
& \pi_{0}\left(R_{n}^{-1}(y)\right)=f_{n}\left(\left(\pi_{1}\left(R_{n}^{-1}(y)\right)\right)_{0}\right) \& \\
& \left.\left(\left(\pi_{1}\left(R_{n}^{-1}(y)\right)\right)_{0},\left(\pi_{1}\left(R_{n}^{-1}(y)\right)\right)_{1}\right) \in A\right] .
\end{aligned}
$$

So $g^{-1}$ is also $C$-measurable.

Finally, $g(x, \alpha) F_{n(x)} f_{n(x)}(x) F_{n(x)} x$, so $g(x, \alpha) E x$.

Proof of Lemma 3.7. Clearly $R_{0}=\varnothing$. By a standard result of descriptive set theory, we can find $R_{1}^{*}$ satisfying the conditions of the lemma for $F_{1}$. Put $R_{1}(x, \alpha)=$ $R_{1}^{*}(x,\langle 1, \alpha\rangle)$, where \langle\rangle is a Borel bijection of $\mathbb{N} \times 2^{\mathbb{N}}$ with $2^{\mathbb{N}}$. This works as well.

Now consider $A_{2}$ and split it in two parts: $A_{2}^{\prime}=\left\{x \in A_{2}: \exists y\left[y \in[x]_{F_{2}} \&[y]_{F_{1}}\right.\right.$ is uncountable\}, $A_{2}^{\prime \prime}=A_{2} \backslash A_{2}^{\prime}$. So $A_{2}^{\prime}$ is $\boldsymbol{\Sigma}_{1}^{1}$ and $A_{2}^{\prime \prime}$ is in $\boldsymbol{\Pi}_{1}^{1} \& \boldsymbol{\Sigma}_{1}^{1}$, so certainly both are $C$-measurable. Let $g_{2}$ be a $C$-measurable function with domain $A_{2}^{\prime}$, such that $g_{2}(x)$ is a $y$ witnessing that $x \in A_{2}^{\prime}$. Let $f_{1}(y)=z \in T_{1}$. Thus $[z]_{F_{1}}$ is uncountable. Put $R_{2}^{* *}(x, \alpha)=R_{1}^{*}(z,\langle 2, \alpha\rangle)$. Then $R_{2}^{* *}$ is a $C$-measurable bijection with domain $A_{2}^{\prime}$. (Note that given $z, x$ can be determined as $x=f_{2}(z)$.) Let $R_{2}^{* * *}$ be a $C$-measurable injection with domain $A_{2}^{\prime \prime}$ satisfying (i) of the lemma for $x \in A_{2}^{\prime \prime}$, $n=2$. Put $R_{2}^{*}=R_{2}^{* *} \cup R_{2}^{* * *}$. Clearly $R_{2}^{*}$ satisfies all the conditions of the lemma for $F_{2}$. Put

$$
R_{2}(x, \alpha)=R_{2}^{*}(x,\langle 1, \alpha\rangle) .
$$

Next, split $A_{3}$ into two parts: $A_{3}^{\prime}=\left\{x \in A_{3}: \exists y\left[y \in[x]_{F_{2}} \&[y]_{F_{2}}\right.\right.$ is uncountable]\}, $A_{3}^{\prime \prime}=A_{3} \backslash A_{3}^{\prime}$. For $A_{3}^{\prime \prime}$, define $R_{3}^{* * *}$ as before. For $A_{3}^{\prime}$, let $g_{3}$ be $C$-measurable choosing a witness $y=g_{3}(x)$ to the fact that $x \in A_{3}^{\prime}$. Let $f_{2}(y)=z \in T_{2}$. Thus $z \in A_{2}$. If $z \in A_{2}^{\prime \prime}$, let $R_{3}^{* *}(x, \alpha)=R_{2}^{* * *}(z,\langle 2, \alpha\rangle)$. If $z \in A_{2}^{\prime}$, let $f_{1}\left(g_{2}(z)\right)=w \in T_{1}$ 
and let $R_{3}^{* *}(x, \alpha)=R_{1}^{*}(w,\langle 3, \alpha\rangle)$. Finally, put $R_{3}^{*}=R_{3}^{* *} \cup R_{3}^{* * *}$ and $R_{3}(x, \alpha)=$ $R_{3}^{*}(x,\langle 1, \alpha\rangle)$.

Proceed this way by induction on $n$.

We conclude this section with an additional result that further clarifies the role of strong hypersmoothness.

Theorem 3.8. If the Borel equivalence relation $E$ is smooth and strongly hypersmooth, then $E$ is strongly smooth.

Remark. Note that this also implies that the assumption that $E$ is strongly hypersmooth is essential in 3.3, because by 3.8 (and the remarks preceding 3.1) there is a smooth but not strongly hypersmooth $E$. Then $E \times I_{2^{\mathbb{N}}} \sqsubseteq^{i} E_{1}$ fails, since otherwise $E \times I_{2^{\mathbb{N}}}$ would be strongly hypersmooth, as $E_{1}$ is, and so would be $E$.

Proof (of 3.8). By relativization, it is enough to assume that $E$ is a $\Delta_{1}^{1}$ equivalence relation on $\mathcal{N},\left(E_{n}\right)$ is a $\Delta_{1}^{1}$-sequence of $\Pi_{1}^{0}$ equivalence relations on $\mathcal{N}$ with $E_{0} \subseteq$ $E_{1} \subseteq \cdots$ and $\bigcup_{n} E_{n}=E, f: \mathcal{N} \rightarrow \mathcal{N}$ is $\Delta_{1}^{1}$ such that $x E y \Leftrightarrow f(x)=f(y)$, and $\left(f_{n}\right)$ is a $\Delta_{1}^{1}$-sequence of $\Delta_{1}^{1}$ functions $f_{n}: \mathcal{N} \rightarrow \mathcal{N}$ so that $f_{n}$ is a selector for $E_{n}$. Let $f(\mathcal{N})=A$, which is a $\Sigma_{1}^{1}$ subset of $\mathcal{N}$. To show that $E$ is strongly smooth, it is enough to show that for each $z \in A$ there is $x \in \Delta_{1}^{1}(z)$ with $f(x)=z$.

Let $C=f^{-1}[\{z\}]$. If $y \in C$, then $[y]_{E}=C$, so $C=\bigcup_{n}[y]_{E_{n}}$. Apply the Baire Category Theorem in the Gandy-Harrington topology relativized to $z$ (i.e., the topology $\tau^{z}$ whose basic nbhds are the $\Sigma_{1}^{1}(z)$ subsets of $\left.\mathcal{N}\right)$, noticing that $C \in \tau^{z}$ and $[y]_{E_{n}}$ are closed in $\mathcal{N}$, so $\tau^{z}$-closed. We can then find $S \subseteq[y]_{E_{n}}$, for some $n$, with $S \neq \varnothing, S \in \Sigma_{1}^{1}(z)$. Now $f_{n}\left(x^{\prime}\right)=f_{n}(y), \forall x^{\prime} \in S$, so $x=f_{n}(y) \in \Delta_{1}^{1}(z) \cap C$ and we are done.

\section{EMBEDDing $E_{1}$}

It is a delicate question to decide, for a given Borel equivalence relation $E$, whether $E_{1} \leq E$. The only obstruction we know is given in 4.1 below.

Let $E$ be a Borel equivalence relation on $X$. We call $E$ idealistic if there is a map $C \in X / E \mapsto I_{c}$, assigning to each $E$-equivalence class $C$ a $\sigma$-ideal $I_{C}$ of subsets of $C$, with $C \notin I_{C}$, such that $I_{C}$ satisfies the ccc (countable chain condition), i.e., any collection of pairwise disjoint subsets of $C$ which are not in $I_{C}$ is countable, and moreover the map $C \mapsto I_{C}$ is Borel in the following sense:

For each Borel $A \subseteq X^{2}$ the set $A_{I}$ defined by

$$
x \in A_{I} \Leftrightarrow\left\{y \in[x]_{E}: A(x, y)\right\} \in I_{[x]_{E}}
$$

is Borel.

Examples of idealistic $E$ include those induced by Borel actions of Polish groups and the measured ones, i.e., those for which there is a Borel assignment $x \mapsto \mu_{x}$ of probability measures such that $\mu_{x}\left([x]_{E}\right)=1$ and $x E y \Rightarrow \mu_{x} \sim \mu_{y}$ (see for example $[\mathrm{K} 1])$.

Theorem 4.1. Let $E$ be an idealistic Borel equivalence relation. Then $E_{1} \not \leq E$.

Proof. If $E_{1} \leq E$, then by $2.10, E_{1} \sqsubseteq^{i} E$, so $E_{1}$ must be idealistic too. But $E_{1}$ is the union of a sequence of smooth Borel equivalence relations, so by Theorem 1.5 of [K1], $E_{1} \leq F$ for a countable Borel equivalence relation $F$, contradicting 1.4. 
If $G$ is a Polish group acting in a Borel way on a standard Borel space $X$ and $E_{G}$ is the corresponding equivalence relation, then $E_{1} \not \leq E_{G}$ by 4.1 provided $E_{G}$ is Borel. However by a modification of the proof of 4.1 we do not need to impose this restriction.

Theorem 4.2. Let $E_{G}$ be the equivalence relation induced by a Borel action of a Polish group. Then $E_{1} \not \leq E_{G}$.

Proof. Assume $E_{1} \leq E_{G}$ via the Borel function $f:\left(2^{\mathbb{N}}\right)^{\mathbb{N}} \rightarrow X$, where $E_{G}$ lives on $X$. Let $f\left(\left(2^{\mathbb{N}}\right)^{\mathbb{N}}\right)=Y$ and $Z=[Y]_{E_{G}}$, so that $Y, Z$ are $\boldsymbol{\Sigma}_{1}^{1}$.

Let $g: Y \rightarrow\left(2^{\mathbb{N}}\right)^{\mathbb{N}}$ be a $C$-measurable inverse for $f$ and define the equivalence relation $F$ on $Y$ by

$$
y F y^{\prime} \Leftrightarrow g(y) E_{1} g\left(y^{\prime}\right)\left(\Leftrightarrow y E_{G} y^{\prime}\right) .
$$

Then $F=\bigcup_{n} F_{n}$, where $F_{0} \subseteq F_{1} \subseteq \cdots$ are equivalence relations on $Y$ which are $C$-measurable smooth, i.e., for each $n$ there is a $C$-measurable function $s_{n}: Y \rightarrow 2^{\mathbb{N}}$ with $y F_{n} y^{\prime} \Leftrightarrow s_{n}(y)=s_{n}\left(y^{\prime}\right)$.

Now for each $D \in X / E_{G}$ let $I_{D}$ be the canonical $\sigma$-ideal on $D$ (see, e.g., [K1]) given by

$$
A \in I_{D} \Leftrightarrow\{g \in G: g \cdot x \in A\} \text { is meager in } G,
$$

where $(g, x) \mapsto g \cdot x$ is the action and $x \in D$. (This definition is independent of $x$.) Clearly $I_{D}$ is ccc. Fix a $C$-measurable function $h: Z \rightarrow Y$ such that $h(z) E_{G} z$. For each $C \in Y / F$, define the following $\sigma$-ideal $J_{C}$ on $C$ :

$$
A \in J_{C} \Leftrightarrow h^{-1}(A) \in I_{[C]_{E_{G}}} .
$$

It is clearly ccc, and $C \notin J_{C}$. Also $C \mapsto J_{C}$ satisfies the following:

For each $C$-measurable $C \subseteq Y^{2}$, the set $C_{J}$ defined by

$$
y \in C_{J} \Leftrightarrow\left\{y^{\prime} \in[y]_{F}: C\left(y, y^{\prime}\right)\right\} \in J_{[y]_{F}}
$$

is $\boldsymbol{\Delta}_{2}^{1}$.

We can now repeat the argument for the proof of 1.5 , (ii) $\Rightarrow$ (i) in [K1], to show that there is a $\boldsymbol{\Sigma}_{2}^{1}$ set $A \subseteq Y$ which meets every $F$-equivalence class in a countable nonempty set. Let $F^{\prime}=F \mid A$. It follows that there is a $\Delta_{2}^{1}$ function $H:\left(2^{\mathbb{N}}\right)^{\mathbb{N}} \rightarrow X$ such that $x E_{1} y \Leftrightarrow H(x) F^{\prime} H(y)$. Then we can repeat the proof of 1.5 (with $H$ replacing $f$ there) to reach a contradiction. The only additional fact that is needed in the present case is that $H$ is Baire measurable, i.e., we need to know that $\Sigma_{2}^{1}$ sets have the Baire property. However, since the result we want to prove (i.e., 4.2) is equivalent (in ZFC) to a $\Pi_{3}^{1}$ sentence, it is enough, by standard metamathematical results, to prove it assuming additionally $\mathrm{MA}+\neg \mathrm{CH}$, which implies that all $\boldsymbol{\Sigma}_{2}^{1}$ sets have the property of Baire, and we are done.

We can use these results to discuss various classes of examples. Let us consider first equivalence relations generated by filters on $\mathbb{N}$; see $[\mathrm{L}]$. For $E$ a Borel equivalence relation on $X$ and $\mathcal{F}$ a Borel filter on $\mathbb{N}$, denote by $E^{\mathcal{F}}$ the following Borel equivalence relation on $X^{\mathbb{N}}$ :

$$
\left(x_{n}\right) E^{\mathcal{F}}\left(y_{n}\right) \Leftrightarrow\left\{n: x_{n} E y_{n}\right\} \in \mathcal{F} .
$$

If $E=\Delta_{2}$ is the equality relation on $2=\{0,1\}$, we write $2^{\mathcal{F}}$ instead of $\Delta_{2}^{\mathcal{F}}$.

If $E$ has uncountably many equivalence classes, then $\Delta_{2^{\mathbb{N}}} \leq E$, so $E_{1}=\Delta_{2^{\mathbb{N}}}^{\mathcal{N}_{0}} \leq$ $E^{\mathcal{N}_{0}}$, where $\mathcal{N}_{0}=$ the Fréchet filter $=\{A \subseteq \mathbb{N}: A$ is cofinite $\}$. Given two filters 
$\mathcal{F}, \mathcal{G}$, let $\mathcal{F} \leq \mathcal{G}$ iff there is $\varphi: \mathbb{N} \rightarrow \mathbb{N}$ with $\mathcal{F}=\varphi \mathcal{G}=\left\{A \subseteq \mathbb{N}: \varphi^{-1}[A] \in \mathcal{G}\right\}$. It is well-known that if $\mathcal{F}$ is a free Borel filter, then $\mathcal{N}_{0} \leq \mathcal{F}$, and it is easy to see that

$$
\mathcal{F} \leq \mathcal{G} \Rightarrow E^{\mathcal{F}} \leq E^{\mathcal{G}} .
$$

So $E^{\mathcal{N}_{0}} \leq E^{\mathcal{F}}$ for any free Borel $\mathcal{F}$, and thus for any Borel $E$ with uncountably many equivalence classes we have $E_{1} \leq E^{\mathcal{F}}$.

The situation with $2^{\mathcal{F}}$ is quite different. Clearly $E_{1} \not \leq 2^{\mathcal{N}_{0}}=E_{0}$. We denote by $\mathbb{N}$ also the filter $\{\mathbb{N}\}$. Then $E_{1} \cong 2^{\mathbb{N} \times \mathcal{N}_{0}}$, so $E_{1} \leq 2^{\mathcal{F}}$ for any $\mathcal{F}$ with $\mathbb{N} \times \mathcal{N}_{0} \leq \mathcal{F}$. Since $\mathbb{N} \leq \mathcal{N}_{0}$, it follows that $\mathbb{N} \times \mathcal{N}_{0} \leq \mathcal{N}_{0} \times \mathcal{N}_{0}=\mathcal{N}_{1}$ and so $E_{1} \leq 2^{\mathcal{N}}$ and thus $E_{1} \leq 2^{\mathcal{N}_{\xi}}$, where $\mathcal{N}_{\xi}$ are the iterated Fréchet filters; see [L].

On the other hand, every ideal on $\mathbb{N}$ is also a subgroup of the compact group $\left(\mathbb{Z}_{2}^{\mathbb{N}},+\right)$, where + is coordinatewise addition. If $\mathcal{F}$ is a Borel filter and $\mathcal{I}$ its dual ideal, then $\mathcal{I}$ is a Borel subgroup of $\mathbb{Z}_{2}^{\mathbb{N}}$ and $2^{\mathcal{F}}$ is exactly the Borel equivalence relation given by the cosets of $\mathcal{I}$ in $\mathbb{Z}_{2}^{\mathbb{N}}$, thus is generated by a Borel $\mathcal{I}$-action. Recall now that a standard Borel group $G$ (i.e., a group which is a standard Borel space for which multiplication and inverse are Borel) is called Polishable if there is a (necessarily unique) Polish topology on $G$ with the same Borel structure, under which $G$ becomes a topological group. Thus if $\mathcal{I}$ is Polishable, $E_{1} \not \leq 2^{\mathcal{F}}$ by 4.1. This has an interesting application concerning ideals:

If $\mathcal{I}$ is a Borel ideal, $\mathcal{F}$ its dual filter and $\mathbb{N} \times \mathcal{N}_{0} \leq \mathcal{F}$, then $\mathcal{I}$ is not Polishable.

On the other hand, there are interesting Polishable ideals $\mathcal{I}$ (for which therefore $\left.E_{1} \not \leq 2^{\mathcal{F}}\right)$. For instance, let $k_{0}=0<k_{1}<k_{2}<\cdots$ be such that $k_{n+1}=k_{n}+n$ and let $I_{n}=\left[k_{n}, k_{n+1}\right)$, so that $\operatorname{card}\left(I_{n}\right)=n$. Define, for $p \in[1, \infty)$, the ideal $\mathcal{I}_{p}$ by

$$
A \in \mathcal{I}_{p} \Leftrightarrow\left(\operatorname{card}\left(A \cap I_{n}\right) / n\right)_{n \geq 1} \in l^{p} .
$$

Another class of $E$ for which $E_{1} \not \leq E$ comes from model theory. If $E$ is the isomorphism relation on a Borel (invariant under isomorphism) class of countable structures, then by $4.2, E_{1} \not \leq E$. In particular, this applies to the examples discussed in [FS]. For each Borel equivalence relation $E$ on $X$, we denote, as in [L], by $E^{+}$the following equivalence relation on $X^{\mathbb{N}}$ :

$$
\left(x_{n}\right) E^{+}\left(y_{n}\right) \Leftrightarrow \forall n \exists m\left(x_{n} E y_{m}\right) \& \forall n \exists m\left(x_{m} E y_{n}\right) .
$$

Then $\Delta_{2^{\mathbb{N}}}^{+}, \Delta_{2^{\mathbb{N}}}^{++}, \ldots$ (to the transfinite) can be Borel reduced to the equivalence relation of isomorphism of trees discussed in [FS], so $E_{1}$ embeds in none of them either.

Another important Borel equivalence relation is measure equivalence $\sim$. (Here $X$ is the space of probability measures on an uncountable standard Borel space, and $\mu \sim \nu \Leftrightarrow \mu \ll \nu \& \nu \ll \mu$.) By the Spectral Theorem, $\sim \leq E$, where $E$ is the equivalence relation of unitary isomorphism of normal operators on Hilbert space. By $4.2, E_{1} \not \leq E$, so $E_{1} \not \leq \sim$. We can reduce to $\sim$ various other Borel equivalence relations and thus use this to give alternative proofs that $E_{1}$ cannot be reduced to them. For example, consider $E_{C}=\Delta_{2^{\mathbb{N}}}^{+} \leq \sim$ (use $\left(x_{n}\right) \mapsto \sum 2^{-n} \delta_{x_{n}}$, with $\delta_{x}=$ the Dirac measure on $x$ ). Now if $F$ is a countable Borel equivalence relation, then $F \leq E_{C}$ (send any $x$ to an enumeration of $[x]_{F}$ in a Borel way) and easily $E_{C}^{\mathbb{N}} \leq E_{C}$. It follows that $E_{1} \not \leq E_{C}$ and $E_{1} \not \leq F^{\mathbb{N}}$, for any countable $F$.

Finally, note that in [K1] an example is given of a $K_{\sigma}$ subgroup $H$ of $\mathbb{T}^{\mathbb{N}}$ which is Polishable but $E_{H}$ is not comparable in $\leq$ with $E_{1}$. Another example would be $\mathcal{I}_{p}$. So 3.5 does not extend to $F_{\sigma}$ subgroups. 
We conclude with the following problems:

Problem. If $E$ is a Borel equivalence relation, is it true that either $E_{1} \leq E$ or $E$ is idealistic?

Problem. Let $\mathcal{F}$ be a Borel filter on $\mathbb{N}$ and $\mathcal{I}$ its dual ideal. Is it true that either $E_{1} \leq 2^{\mathcal{F}}$ or $\mathcal{I}$ is Polishable? (This has been recently solved affirmatively by Solecki.)

\section{Global effects}

Although the preceding results are "local", being concerned with Borel equivalence relations which are $\leq E_{1}$, they have a surprising "global" consequence about the structure of the class of all Borel equivalence relations.

Given a pair $\left(E, E^{*}\right)$ of Borel equivalence relations with $E<E^{*}$, we say that $\left(E, E^{*}\right)$ satisfies the dichotomy property if for any Borel equivalence relation $F$ we have $F \leq E$ or $E^{*} \leq F$.

Thus Silver's Theorem (see $[\mathrm{S}])$ asserts that $\left(\Delta_{\mathbb{N}}, \Delta_{2^{\mathbb{N}}}\right)$ satisfies the dichotomy property, and the Glimm-Effros type dichotomy proved in [HKL] implies that $\left(\Delta_{2^{\mathbb{N}}}, E_{0}\right)$ satisfies the dichotomy property. Notice also that trivially $\left(\Delta_{n}, \Delta_{n+1}\right)$ $(n=1,2, \ldots)$ satisfy the dichotomy property. If $\left(E, E^{*}\right)$ satisfies the dichotomy property, then $E, E^{*}$ are nodes in $\leq$, i.e., every Borel equivalence relation is comparable in $\leq$ to each one of them.

We have, now,

Theorem 5.1. Let $E$ be a Borel equivalence relation which is a node for $\leq$, i.e., for any Borel equivalence relation $F$ we have $E \leq F$ or $F \leq E$. Then $E \approx^{*} \Delta_{n}$ $(n=1,2, \ldots), \Delta_{\mathbb{N}}, \Delta_{2^{\mathbb{N}}}$ or $E_{0}$.

In particular, the only pairs $\left(E, E^{*}\right)$ satisfying the dichotomy property are $\left(\Delta_{n}, \Delta_{n+1}\right)(n=1,2, \ldots),\left(\Delta_{\mathbb{N}}, \Delta_{2^{\mathbb{N}}}\right),\left(\Delta_{2^{\mathbb{N}}}, E_{0}\right)$.

Proof. Call a class $U$ of Borel equivalence relations unbounded (in $\leq$ ) if there is no Borel equivalence relation $E$ such that $\forall F \in U(F \leq E)$. We will use the following result.

Theorem 5.2 (Harrington, unpublished). There is a nonempty class $U$ of Borel equivalence relations which is unbounded, and every $F \in U$ is induced by a Borel action of a Polish group (so in particular is idealistic).

Let $E$ be a Borel equivalence relation which is such that $E \leq F$ or $F \leq E$ for all Borel equivalence relations $F$. By applying 5.2, we obtain that $E \leq F$ for some $F \in U$. We also have $E_{1} \leq E$ or $E \leq E_{1}$. If $E_{1} \leq E$ then $E_{1} \leq F$, which violates 4.1, so $E \leq E_{1}$. Then by 2.1, and the remarks following it, either $E \approx^{*} E_{1}$, which is impossible, or $E \approx^{*} E_{0}$ or $E$ is smooth. In the last case, clearly (by Silver's Theorem) $E \approx^{*} \Delta_{2^{\mathbb{N}}}$ or $E \leq E_{\mathbb{N}}$, and if $E \leq E_{\mathbb{N}}$, either $E \approx^{*} E_{n}, n=1,2, \ldots$, or $E \approx \approx^{*} E_{\mathbb{N}}$.

\section{REFERENCES}

[DJK] R. Dougherty, S. Jackson and A. S. Kechris, The structure of hyperfinite Borel equivalence relations, Trans. Amer. Math. Soc., 341 (1), 193-225, 1994. MR 94c:03066

[FR] J. Feldman and A. Ramsey, Countable sections for free actions of groups, Adv. Math., 55, 224-227, 1985. MR 86h:28011

[FS] H. Friedman and L. Stanley, A Borel reducibility theory for classes of countable structures, J. Symb. Logic, 54 (3), 894-914, 1989. MR 91f:03062 
[HKL] L. Harrington, A. S. Kechris and A. Louveau, A Glimm-Effros dichotomy for Borel equivalence relations, J. Amer. Math. Soc., 3 (4), 903-927, 1990. MR 91h:28023

[HMS] L. Harrington, D. Marker and S. Shelah, Borel orderings, Trans. Amer. Math. Soc., 310, 292-302, 1988. MR 90c:03041

[JKL] S. Jackson, A. S. Kechris and A. Louveau, On countable Borel equivalence relations, to appear.

[K1] A. S. Kechris, Countable sections for locally compact group actions, Ergod. Th. \& Dynam. Sys., 12, 283-295, 1992. MR 94b:22003

[K2] - Countable sections for locally compact group actions, II, Proc. Amer. Math. Soc., 120, 241-247, 1994. MR 94b:22004

[K3] Classical Descriptive Set Theory, Springer-Verlag, New York, 1995. MR 96e:03057

[L] A. Louveau, On the reducibility order between Borel equivalence relations, Stud. Logic Found. Math., vol. 134, North-Holland, Amsterdam, 1994. MR 96g:03081

[Mi] D. Miller, On the measurability of orbits in Borel actions, Proc. Amer. Math. Soc., 63, 165-170, 1977. MR 55:13394

[Mo] Y. N. Moschovakis, Descriptive Set Theory, North Holland, Amsterdam, 1980. MR 82e:03002

[R] J. T. Rogers, Jr., Borel transversals and ergodic measures on indecomposable continua, Topology and its Appl., 24, 217-227, 1986. MR 88a:54078

[S] J. H. Silver, Counting the number of equivalence classes of Borel and coanalytic equivalence relations, Ann. Math. Logic. 18, 1-28, 1980. MR 81d:03051

[V] A. M. Vershik, Trajectory Theory, in Ya. G. Sinai (Ed.), Dynamical Systems II, SpringerVerlag, 77-98, 1989. MR 91i:58079

[VF] A. M. Vershik and A. L. Fedorov, Trajectory Theory, J. Soviet Math., 38 (2), 1799-1822, 1987. MR 88b:28031

[VG] V. G. Vinokurov and N. N. Ganikhodzhaev, Conditional functions in the trajectory theory of dynamical systems, Math. USSR Izvestija, 13 (2), 221-252, 1979. MR 80d:28038

[W] V. M. Wagh, A descriptive version of Ambrose's representation theorem for flows, Proc. Ind. Acad. Sci. (Math. Sci.), 98, 101-108, 1988. MR 90m:28021

Department of Mathematics, A. P. Sloan Laboratory of Mathematics and Statistics, California Institute of Technology, Pasadena, California 91125

E-mail address: kechris@caltech.edu

Equipe D'Analyse, Université Paris Vi, 4, Place Jussieu, 75230 Paris Cedex 05, France

E-mail address: louveau@ccr.jussieu.edu 